\title{
Lyndon words and transition matrices between elementary, homogeneous and monomial symmetric functions
}

\author{
Andrius Kulikauskas* \\ Minciu Sodas Laboratory \\ Vilnius, Lithuania \\ La Jolla, CA 92093-0112. USA \\ ms@ms. It
}

\author{
Jeffrey Remmel ${ }^{\dagger}$ \\ Department of Mathematics \\ University of California, San Diego \\ La Jolla, CA 92093-0112. USA \\ jremmel@ucsd.edu
}

Submitted: Jun 23, 2004; Accepted: Feb 22, 2006; Published: Feb 28, 2006

MR Subject Classification: 05E05,05A99

\begin{abstract}
Let $h_{\lambda}, e_{\lambda}$, and $m_{\lambda}$ denote the homogeneous symmetric function, the elementary symmetric function and the monomial symmetric function associated with the partition $\lambda$ respectively. We give combinatorial interpretations for the coefficients that arise in expanding $m_{\lambda}$ in terms of homogeneous symmetric functions and the elementary symmetric functions. Such coefficients are interpreted in terms of certain classes of bi-brick permutations. The theory of Lyndon words is shown to play an important role in our interpretations.
\end{abstract}

\section{Introduction}

Let $\Lambda_{n}$ denote the space of homogeneous symmetric functions of degree $n$ in infinitely many variables $x_{1}, x_{2}, \ldots$. There are six standard bases of $\Lambda_{n}:\left\{m_{\lambda}\right\}_{\lambda \vdash n}$ (the monomial symmetric functions), $\left\{h_{\lambda}\right\}_{\lambda \vdash n}$ (the complete homogeneous symmetric functions), $\left\{e_{\lambda}\right\}_{\lambda \vdash n}$ (the elementary symmetric functions), $\left\{p_{\lambda}\right\}_{\lambda \vdash n}$ (the power symmetric functions), $\left\{s_{\lambda}\right\}_{\lambda \vdash n}$ (the Schur functions) and $\left\{f_{\lambda}\right\}_{\lambda \vdash n}$ (the forgotten symmetric functions) where $\lambda \vdash n$ denotes that $\lambda$ is a partition of $n$. We let $\ell(\lambda)$ denote the length of $\lambda$, i.e. $\ell(\lambda)$ equals the number of parts of $\lambda$. The entries of the transition matrices between these bases of symmetric functions all have combinatorial significance. For example, Doubilet [2] showed that all such entries could be interpreted via the lattice of set partitions $\pi_{n}$ and

${ }^{*}$ The authors would like to thank the anonymous referee who suggested numerous improvements for the presentation of this paper.

${ }^{\dagger}$ Supported in part by NSF grant DMS 0400507 
its Möbius function. More recently, Beck, Remmel, and Whitehead [1] gave a complete list of combinatorial interpretations of such entries.

The main purpose of this paper is to provide proofs for two of the combinatorial interpretations described in [1] that have not previously been published, namely, the entries of the transition matrices which allow one to express the monomial symmetric function $m_{\mu}$ in terms of the homogeneous symmetric functions $h_{\lambda}$ and the elementary symmetric functions $e_{\lambda}$.

More formally, given two bases of $\Lambda_{n},\left\{a_{\lambda}\right\}_{\lambda \vdash n}$ and $\left\{b_{\lambda}\right\}_{\lambda \vdash n}$, we fix some standard ordering of the set of partitions of $n$, such as the lexicographic order, and then we think of the bases as row vectors, $\left\langle a_{\lambda}\right\rangle_{\lambda \vdash n}$ and $\left\langle b_{\lambda}\right\rangle_{\lambda \vdash n}$. We define the transition matrix $M(a, b)$ by the equation

$$
\left\langle b_{\lambda}\right\rangle_{\lambda \vdash n}=\left\langle a_{\lambda}\right\rangle_{\lambda \vdash n} M(a, b) .
$$

Thus $M(a, b)$ is the matrix that transforms the basis $\left\langle a_{\lambda}\right\rangle_{\lambda \vdash n}$ into the basis $\left\langle b_{\lambda}\right\rangle_{\lambda \vdash n}$ and the $(\lambda, \mu)$-th entry of $M(a, b)$ is defined by the equation

$$
b_{\mu}=\sum_{\lambda \vdash n} a_{\lambda} M(a, b)_{\lambda, \mu} .
$$

We note that our convention for the transition matrix $M(a, b)$ differs from that of Macdonald [6] since Macdonald interprets $\left\langle a_{\lambda}\right\rangle_{\lambda \vdash n}$ as a column vector.

The goal of this paper is to give combinatorial interpretations for $M(h, m)_{\lambda, \mu}$ and $M(e, m)_{\lambda, \mu}$. To describe our interpretations of $M(h, m)_{\lambda, \mu}$ and $M(e, m)_{\lambda, \mu}$, we must first introduce the concept of a primitive bi-brick permutation. Given partitions $\lambda=$ $\left(\lambda_{1}, \ldots, \lambda_{\ell}\right)$ and $\mu=\left(\mu_{1}, \ldots, \mu_{k}\right)$ of $n$, define a $(\lambda, \mu)$-bi-brick permutation as follows. We shall consider cycles $C$ which are nothing more than circles which are partitioned in $s$ equal arcs or cells for some $s \geq 1$. The length, $|C|$, of any such cycle $C$ is defined to be the number of cells of $C$. Let $C_{1}, C_{2}, \ldots, C_{t}$ be a multiset of cycles whose lengths sum to $n$. Assume we have a set of bricks of sizes $\lambda_{1}, \ldots, \lambda_{\ell}$ called $\lambda$-bricks and a set of bricks of size $\mu_{1}, \ldots, \mu_{k}$ called $\mu$-bricks. On each cycle, place an outer tier of $\lambda$-bricks and an inner tier of $\mu$-bricks whose lengths sum to the length of the cycle. The resulting set of bi-brick cycles will be called a $(\lambda, \mu)$-bi-brick permutation. If the bricks are placed in such a way that no cycle has rotational symmetry, then the bi-brick permutation is called primitive. For example, suppose $\lambda=\left(2^{5}\right), \mu=\left(1^{2}, 2^{4}\right)$, and $C_{1}=4, C_{2}=4$, and $C_{3}=2$. Figure 1(a) shows a $(\lambda, \mu)$-bi-brick permutation which is not primitive since the first and second cycles have rotational symmetry. Figure $1(\mathrm{~b})$ shows a $(\lambda, \mu)$-bi-brick permutation which is primitive since no cycle has rotational symmetry.

An alternative way to understand the notion of a primitive bi-brick cycle $C$ is to use the theory of Lyndon words. Given an ordered alphabet $X=\left\{x_{1}<\ldots<x_{r}\right\}$, let $X^{*}$ denote the set of all words over the alphabet $X$. We then can use the lexicographic order to give a total ordering to $X^{*}$ by declaring that for two words $w=w_{1} \cdots w_{n}$ and $v=v_{1} \cdots v_{n}, v \leq_{\ell} w$ if and only if either (a) there is an $i \leq \min \{m, n\}$ such that $v_{i}<w_{i}$ and $v_{j}=w_{j}$ for $j<i$ or (b) $m<n$ an $v_{j}=w_{j}$ for all $j \leq m$. We let $\epsilon$ denote the empty word which has length 0 by definition. If $w=w_{1} \cdots w_{s}$, then we say $w$ has length $s$ and write $|w|=s$. We let $X^{+}=X^{*}-\{\epsilon\}$. If $w=w_{1} \cdots w_{s}$ and $v=v_{1} \cdots v_{t}$, then 
$w v=w_{1} \cdots w_{s} v_{1} \cdots v_{t}$. For any word $w$ with $|w| \geq 1$, we define $w^{r}$ for $r \geq 1$ by induction as $w^{1}=w$, and for $r>1, w^{r}=w^{r-1} w$. We say that a nonempty word $w=w_{1} \cdots w_{s}$ is Lyndon if either $s=1$ or $s>1$ and $w$ is the lexicographically least element in its cyclic rearrangement class. For example, if $w=x_{1} x_{2} x_{1} x_{3}$, then the cyclic rearrangement class of $w$ is

$$
\left\{x_{1} x_{2} x_{1} x_{3}, x_{2} x_{1} x_{3} x_{1}, x_{1} x_{3} x_{1} x_{2}, x_{3} x_{1} x_{2} x_{1}\right\}
$$

so that $w$ is Lyndon since it is the lexicographically least element in its set of cyclic rearrangement class. In fact, one can show that if $w$ has length greater than or equal to 2 and $w$ is not Lyndon, then $w=u^{r}$ for some word $u \in X^{+}$and $r \geq 2$, see [5].

We shall associate to each bi-brick cycle a word in the ordered alphabet $A=\{B<$ $L<N<M\}$ as follows. First, read the cycle clockwise and, for each cell of the cycle, record a $B$ if both a $\lambda$-brick and a $\mu$-brick start in the cell, record an $L$ if a $\lambda$-brick starts at the cell and a $\mu$-brick does not, record an $M$ if a $\mu$-brick starts at the cell and a $\lambda$-brick does not, and record an $N$ if neither a $\lambda$-brick nor a $\mu$-brick starts at the cell. We then define the word of the cycle, $W(C)$, to be the lexicographically least circular rearrangement of the cycle of letters associated with $C$. For example, consider the first cycle $C_{1}$ of Figure 1(a). Starting at the top and reading clockwise, the cycle of letters associated with $C_{1}$ is $N B N B=w$. There are just two cyclic rearrangements of $\omega$, namely $N B N B$ and $B N B N$. Since $B N B N$ is the lexicographically least of these two words, $W\left(C_{1}\right)=B N B N$. Below each of the cycles in Figure 1(a) and 1(b), we have listed the word of the cycle. Now if a bi-brick cycle $C$ has rotational symmetry, then $W(C)$ will be a power of a smaller word, i.e. $W(C)=u^{r}$ where $r>1$ and $|u| \geq 1$. Thus a bi-brick cycle $C$ is primitive if $W(C)$ is a Lyndon word. Note that each bi-brick cycle $C$ in a $(\lambda, \mu)$-bi-brick permutation has at least one $\lambda$-brick and at least one $\mu$-brick. Thus $W(C)$ must contain a $B$ if a $\lambda$-brick and $\mu$-brick start at the same cell or, if $W(C)$ contains no $B$, then it must contain both an $L$ and an $M$. Vice versa, it is easy to see that any word $w$ over $A$ such that either (a) $w$ contains a $B$ or (b) $w$ contains no $B$ but $w$ does contain both an $L$ and an $M$ is of the form $W(C)$ for some bi-brick cycle $C$.

We say that a bi-brick permutation is primitive is it consists of entirely of primitive bi-brick cycles. Thus we can think of a primitive bi-brick permutation with $k$ cycles as a multiset $\left\{w_{1} \leq_{\ell} \cdots \leq_{\ell} w_{k}\right\}$ of Lyndon words over A where each $w_{i}$ either contains a $B$ or contains both an $L$ and $M$ if $w_{i} \in\{L, M, N\}^{*}$. Here $\leq_{\ell}$ denotes the lexicographic order on $A^{*}$ relative to ordering of letters $B<L<N<M$. We say a primitive $(\lambda, \mu)$-bibrick permutation is simple if its bi-brick cycles are pairwise distinct. Thus we can think of a simple primitive bi-brick permutation with $k$ cycles as a set $\left\{w_{1}<_{\ell} \cdots<_{\ell} w_{k}\right\}$ of Lyndon words over A where each $w_{i}$ either contains a $B$ or contains both an $L$ and $M$ if $w_{i} \in\{L, M, N\}^{*}$. We let $P B(\lambda, \mu)$ be the set of primitive $(\lambda, \mu)$-bi-brick permutations and $\operatorname{SPB}(\lambda, \mu)$ be the set of simple primitive bi-brick permutations. Define the sign of a bi-brick permutation $\theta, \operatorname{sgn}(\theta)$, to be $(-1)^{n-c}$ where $\lambda, \mu \vdash n$ and $c$ is the number of cycles of $\theta$. This given, the main result of this paper is to prove the following.

Theorem 1 Let $\lambda$ and $\mu$ be partitions of $n$. Then

$$
\text { (i) } M(h, m)_{\lambda, \mu}=(-1)^{\ell(\lambda)+\ell(\mu)}|P B(\lambda, \mu)|
$$


(a)

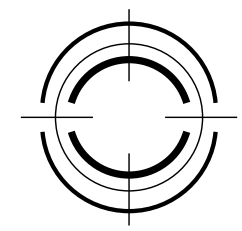

BNBN

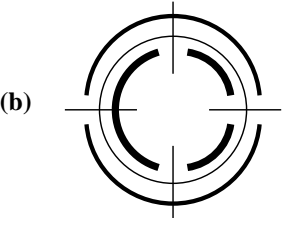

BMLM

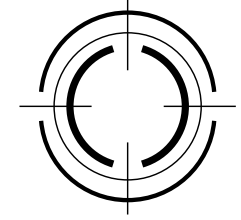

LMLM

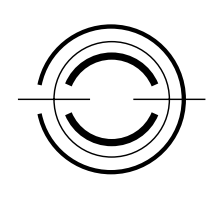

BM

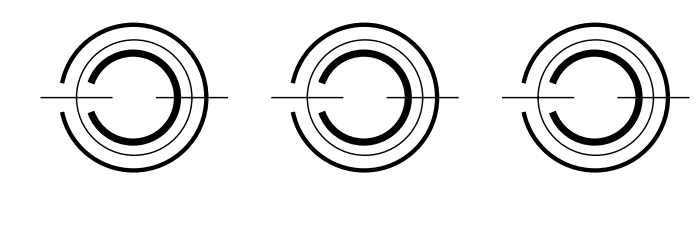

$\mathbf{B N}$

$\mathbf{B N}$

BN

$\begin{aligned} \lambda=\left(2^{5}\right) & \lambda \text {-bricks } \\ \mu=\left(1^{2}, 2^{4}\right) & \mu \text {-bricks }\end{aligned}$

Figure 1: Bi-brick permutations.

and

$$
\text { (ii) } M(e, m)_{\lambda, \mu}=(-1)^{\ell(\lambda)+\ell(\mu)} \sum_{\theta \in S P B(\lambda, \mu)} \operatorname{sgn}(\theta) \text {. }
$$

For example, Figures 2-6 picture all the $(\lambda, \mu)$-brick permutations such that $\lambda=\mu=$ $\left(1^{2}, 2\right)$ where we have partitioned the $(\lambda, \mu)$-bi-brick permutations according to type of the underlying cycles. In Figure 2, we picture the $(\lambda, \mu)$-bi-brick permutations whose cycles induce the partition $(1,1,2)$. We see there are $2(\lambda, \mu)$-bi-brick permutations according to which $(2,2)$-cycles we pick. Neither of the resulting $(\lambda, \mu)$-bi-brick permutations is simple so that the $(\lambda, \mu)$-bi-brick permutations in Figure 2 contribute 2 to $M(h, m)_{\lambda, \mu}$ and 0 to $M(e, m)_{\lambda, \mu}$. In Figure 3, we picture the unique $(\lambda, \mu)$-bi-brick permutation whose cycles induce the partition $(2,2)$ and where one cycle is a $\left(\left(1^{2}\right),(2)\right)$ cycle and the other cycle is a $\left((2),\left(1^{2}\right)\right)$ cycle. It is primitive and simple and has a positive sign so that the bi-brick permutation pictured in Figure 3 contributes 1 to $M(h, m)_{\lambda, \mu}$ and 1 to $M(e, m)_{\lambda, \mu}$. In Figure 4 , we picture the other possibilities for a $(\lambda, \mu)$-bi-brick permutation whose cycles induce the partition $(2,2)$. One can see that the $((1,1),(1,1))$-cycle is not primitive so there is no contribution to either $M(h, m)_{\lambda, \mu}$ or $M(e, m)_{\lambda, \mu}$ in this case. Figure 5 pictures all the possibilities of $(\lambda, \mu)$-bi-brick permutations whose cycles induce the partition $(1,3)$. We see that there are 3 such $(\lambda, \mu)$-bi-brick permutations according to which cycle of type $((1,2)(1,2))$ we pick. All three resulting bi-brick permutations are primitive and simple and have positive sign so that the $(\lambda, \mu)$-bi-brick permutations in Figure 5 contribute 3 to both $M(h, m)_{\lambda, \mu}$ and $M(e, m)_{\lambda, \mu}$. Finally there are $4(\lambda, \mu)$-bi-brick permutations consisting of single cycles which we picture in Figure 6 . We see that these $(\lambda, \mu)$-bi-brick 


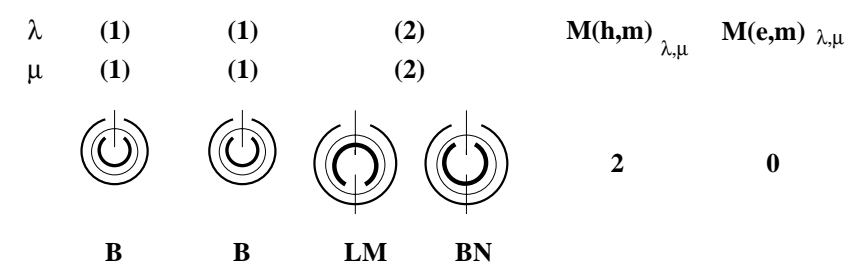

Figure 2: Bi-brick permutations of type $(1,1,2)$.

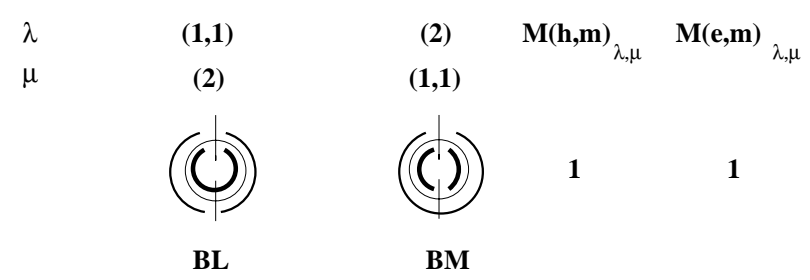

Figure 3: Bi-brick permutations of type $(2,2)$.

permutations all have sign -1 and, hence, they contribute 4 to $M(h, m)_{\lambda, \mu}$ and -4 to $M(e, m)_{\lambda, \mu}$. Thus $M(h, m)_{\left(1^{2}, 2\right),\left(1^{2}, 2\right)}=10$ and $M(e, m)_{\left(1^{2}, 2\right),\left(1^{2}, 2\right)}=0$.

As one can see from figures 2-6, there is considerable cancellation in our expression for $M(e, m)_{\lambda, \mu}$. Thus in section 3, we shall define some sign reversing involutions which will simplify our expression for $M(e, m)_{\lambda, \mu}$. For example, we shall define a sign reversing involution which shows that to compute $M(e, m)_{\lambda, \mu}$, we can restrict ourselves to summing the signs of those simple primitive $(\lambda, \mu)$-bi-brick permutations $\theta$ such that there are at most one cell $c$ where both a $\lambda$-brick and a $\mu$-brick start at $c$ or, equivalently, the number of $B$ 's occuring in the corresponding set of Lyndon words for $\theta$ is $\leq 1$.

We should note that equivalent interpretations for $M(h, m)_{\lambda, \mu}$ and $M(e, m)_{\lambda, \mu}$ first appeared in the first author's thesis [4] although the methods used to find such an interpretation were completely different than the ones presented in this paper.

We note that there are a number of restrictions on the values of $M(h, m)_{\lambda, \mu}$ and

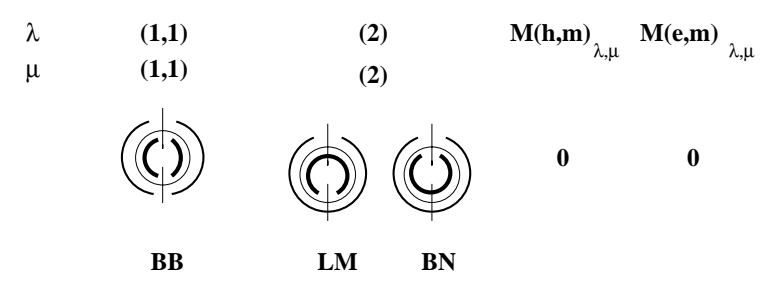

Figure 4: More bi-brick permutations of type $(2,2)$. 


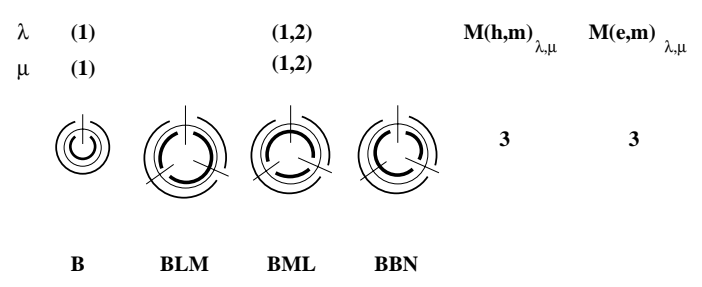

Figure 5: Bi-brick permutations of type $(1,3)$.

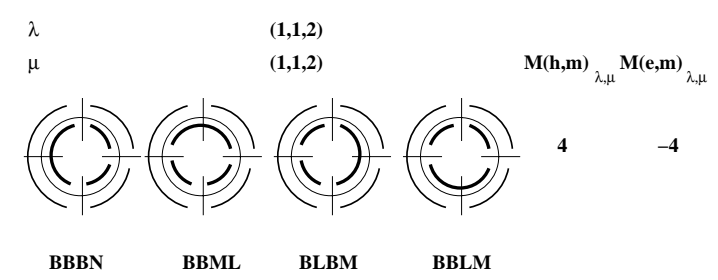

Figure 6: Bi-brick permutations of type (4).

$M(h, m)_{\lambda, \mu}$ that follows from the combinatorial interpretations of well known combinatorial interpretations of the entries of the matrices $M(m, h)$ and $M(m, e)$. That is, suppose $\lambda=\left(\lambda_{1} \geq \cdots \geq \lambda_{k}\right)$ and $\mu=\left(\mu_{1} \geq \cdots \geq \mu_{\ell}\right)$ are partitions of $n$. Then we define the dominance order $\leq_{D}$ on the partitions of $n$ by defining $\lambda \geq_{D} \mu$ if and only if for all $j \leq \max (\{k, \ell\}), \sum_{i=1}^{j} \lambda_{i} \geq \sum_{i=1}^{j} \mu_{i}$. For $k \times \ell$ matrix $M$ with entries from $\mathbb{N}=\{0,1, \ldots\}$, let $r(M)=\left(r_{1}(M), \ldots, r_{k}(M)\right)$ where for each $i, r_{i}(M)=\sum_{j=1}^{\ell} M_{i, j}$ is the $i$-th row sum of $M$. Similarly, let $c(M)=\left(c_{1}(M), \ldots, c_{\ell}(M)\right)$ where for each $i, c_{i}(M)=\sum_{j=1}^{k} M_{j, i}$ is the $i$-th column sum of $M$. Let $\mathbb{N} M_{\lambda, \mu}$ denote the number non-negative integer valued $k \times \ell$ matrices $M$ such that $r(M)=\lambda$ and $c(M)=\mu$ and let $Z_{2} M_{\lambda, \mu}$ denote the number $\{0,1\}$-valued $k \times \ell$ matrices $M$ such that $r(M)=\lambda$ and $c(M)=\mu$. Then

$$
\begin{aligned}
M(m, h)_{\lambda, \mu} & =\mathbb{N} M_{\lambda, \mu} \text { and } \\
M(m, e)_{\lambda, \mu} & =Z_{2} M_{\lambda, \mu},
\end{aligned}
$$

see [6]. It then easily follows that

$$
\begin{aligned}
& M(m, h)_{\lambda, \mu}=M(m, h)_{\mu, \lambda}, \\
& M(m, e)_{\lambda, \mu}=M(m, e)_{\mu, \lambda}, \\
& M(m, e)_{\lambda, \mu} \neq 0 \text { implies } \mu \leq_{D} \lambda^{\prime}, \text { and } \\
& M(m, e)_{\lambda, \lambda^{\prime}}=1,
\end{aligned}
$$

where $\lambda^{\prime}$ denotes the conjugate of $\lambda$, see [6]. Thus $M(m, h)^{T}=M(m, h)$ and $M(m, e)^{T}=$ $M(m, e)$ where for any matrix $M, M^{T}$ denotes the transpose of $M$. It follows that 


$$
\begin{aligned}
M(h, m)^{T}=M(h, m) \text { and } M(e, m)^{T}=M(e, m) \text { so that } \\
M(h, m)_{\lambda, \mu}=M(h, m)_{\mu, \lambda} \\
M(e, m)_{\lambda, \mu}=M(e, m)_{\mu, \lambda} .
\end{aligned}
$$

Note that (11) and (12) also follow from our combinatorial interpretations of $M(h, m)_{\lambda, \mu}$ and $M(e, m)_{\lambda, \mu}$ given in Theorem 1. Finally, let $\prec$ be any total order on partitions which refines the dominance partial order and suppose that $\lambda^{(1)} \prec \cdots \prec \lambda^{(p(n))}$ is the $\prec$-increasing list of all partitions of $n$. Since for all partitions $\lambda$ and $\mu$ of $n, \lambda \leq_{D} \mu$ if and only if $\mu^{\prime} \leq_{D} \lambda^{\prime}$, it follows from (9) and (10) that the $p(n) \times p(n)$ matrix $E=\left\|E_{i, j}\right\|$ where $E_{i, j}=M(m, e)_{\lambda^{(i)},\left(\lambda^{(j)}\right)^{\prime}}$ is an upper triangular matrix with 1's on the diagonal. Thus $E^{-1}=\left\|E_{i, j}^{-1}\right\|$ where $E_{i, j}=M(e, m)_{\left(\lambda^{(i)}\right)^{\prime}, \lambda^{(j)}}$ is also an upper triangular matrix with 1 's on the diagonal and hence

$$
M(e, m)_{\lambda^{\prime}, \mu}=0 \text { if } \mu<_{D} \lambda
$$

and

$$
M(e, m)_{\lambda^{\prime}, \lambda}=1 \text {. }
$$

We also should note that similar results hold for two other transition matrices. Namely, let $\omega: \bigoplus_{n \geq 0} \Lambda_{n} \rightarrow \bigoplus_{n \geq 0} \Lambda_{n}$ be the algebra isomorphism defined by declaring $\omega\left(h_{n}\right)=e_{n}$ for all $n$ where $h_{0}=e_{0}=1$ and $h_{n}=h_{(n)}=\sum_{1 \leq i_{1} \leq \cdots \leq i_{n}} x_{i_{1}} \cdots x_{i_{n}}$ and $e_{n}=e_{(n)}=$ $\sum_{1 \leq i_{1}<\cdots<i_{n}} x_{i_{1}} \cdots x_{i_{n}}$. In [6], it is shown that $\omega$ is an involution and for all partitions $\lambda$, $\omega\left(h_{\lambda}\right)=e_{\lambda}, \omega\left(m_{\lambda}\right)=f_{\lambda}, \omega\left(s_{\lambda}\right)=s_{\lambda^{\prime}}$ and $\omega\left(p_{\lambda}\right)=(-1)^{n-\ell(\lambda)} p_{\lambda}$. It is easy to see that for any bases $\left\{a_{\lambda}\right\}_{\lambda \vdash n}$ and $\left\{b_{\lambda}\right\}_{\lambda \vdash n}$ of $\Lambda_{n}$, the transition matrix from $\left\{\omega\left(a_{\lambda}\right)\right\}_{\lambda \vdash n}$ to $\left\{\omega\left(b_{\lambda}\right)\right\}_{\lambda \vdash n}$ is given by

$$
M(\omega(a), \omega(b))=M(a, b) .
$$

Thus combining Theorem 1 and (15), we have

$$
M(e, f)_{\lambda, \mu}=(-1)^{\ell(\lambda)+\ell(\mu)}|P B(\lambda, \mu)|
$$

and

$$
M(h, f)_{\lambda, \mu}=(-1)^{\ell(\lambda)+\ell(\mu)} \sum_{\theta \in S P B(\lambda, \mu)} \operatorname{sgn}(\theta) .
$$

The outline of this paper is as follows. In section 2 , we shall prove Theorem 1 . In section 3, we shall define a series of involutions which will allow us to give a more refined interpretation of $M(e, m)_{\lambda, \mu}$. That is, we shall show that $M(e, m)_{\lambda, \mu}=(-1)^{\ell(\lambda)+\ell(\mu)}$ $\sum_{\theta \in S P B^{*}(\lambda, \mu)} \operatorname{sgn}(\theta)$ for certain subsets of $\operatorname{SPB}(\lambda, \mu)$. For example, we will show that $S P B^{*}(\lambda, \mu)$ cannot contain any bi-brick permutations $\theta$ such that there are two distinct cells in $\theta$ where both a $\lambda$ and $\mu$ brick start at those cells. These involutions will be defined in terms of our alternative interpretation of primitive bi-brick permutations as sequences of certain Lyndon words and we will heavily use the basic properties of Lyndon words 


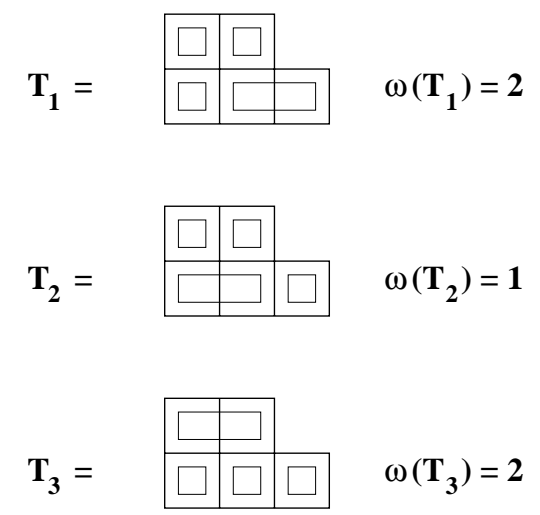

Figure 7: Brick tabloids.

to show that our involutions are well defined. Finally, in section 4, we shall use our interpretations to give the formulas for $M(h, m)_{\lambda, \mu}$ and $M(e, m)_{\lambda, \mu}$ in a number of special cases, In particular, we shall give explicit formulas for $M(h, m)_{\lambda, \mu}$ and $M(e, m)_{\lambda, \mu}$ when $\lambda=\mu=\left(k^{n}\right)$ for some $k$ and $n$, when both $\lambda$ and $\mu$ are two row shapes or when both $\lambda$ and $\mu$ are hook shapes. Finally we shall also give formulas for $M(e, m)_{\lambda, \mu}$ when both $\lambda$ and $\mu$ are two column shapes.

\section{Proof of Theorem 1}

Our proof of Theorem 1 depends on the combinatorial interpretation of the entries of $M(h, p)$ and $M(p, m)$ due to Ĕgecioğlu and Remmel [3]. If $\lambda=\left(\lambda_{1}, \ldots, \lambda_{k}\right)$ is a partition of $n$ which has $\alpha_{i}$ parts of size $i$ for $i=1, \ldots, n$, then we write $\lambda=\left(1^{\alpha_{1}} 2^{\alpha_{2}} \cdots n^{\alpha_{n}}\right)$. This given, we set $z_{\lambda}=1^{\alpha_{1}} 2^{\alpha_{2}} \cdots n^{\alpha_{n}} \alpha_{1} ! \cdots \alpha_{n}$ !. It is well known that $\frac{n !}{z_{\lambda}}=\left|\mathcal{C}_{\lambda}\right|$ where $\mathcal{C}_{\lambda}$ is the set of permutations $\sigma$ of the symmetric group $\mathcal{S}_{n}$ whose cycle lengths induce the partition $\lambda$. A $\lambda$-brick tabloid $T$ of shape $\mu$ is a filling of the Ferrers diagram of $\mu, F_{\mu}$, with $\lambda$-bricks such that (i) each brick lies in a single row of $F_{\mu}$ and (ii) no two bricks overlap. For example, if $\lambda=\left(1^{3}, 2\right)$ and $\mu=(2,3)$, there are three $\lambda$-brick tabloids of shape $\mu$ and these are pictured in Figure 2.

We define the weight of a $\lambda$-brick tabloid $T, \omega(T)$, to be the product of the lengths of the bricks that are at the ends of the rows of $T$. Let $\mathcal{B}_{\lambda, \mu}$ denote the set of $\lambda$-brick tabloids of shape $\mu$ and let

$$
\omega\left(B_{\lambda, \mu}\right)=\sum_{T \in \mathcal{B}_{\lambda, \mu}} \omega(T) .
$$

Then Ĕ̆gecioğlu and Remmel [3] proved the following.

$$
\begin{aligned}
M(h, p)_{\lambda, \mu} & =(-1)^{\ell(\lambda)+\ell(\mu)} \omega\left(B_{\lambda, \mu}\right), \\
M(e, p)_{\lambda, \mu} & =(-1)^{n-\ell(\lambda)} \omega\left(B_{\lambda, \mu}\right),
\end{aligned}
$$




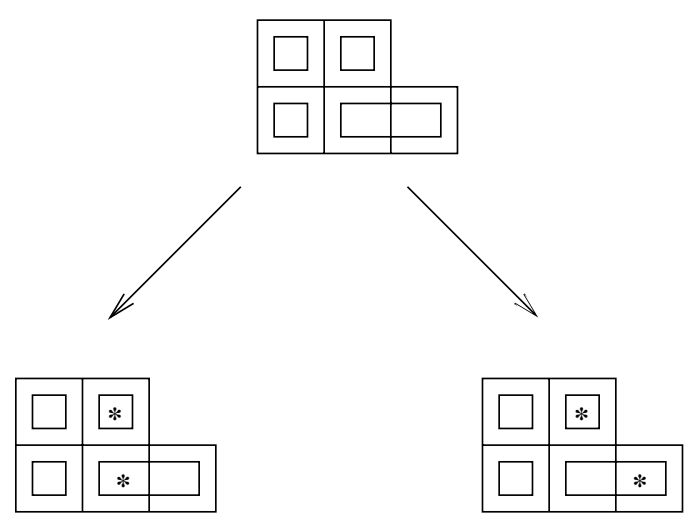

Figure 8: Elements of $B_{\left(1^{3}, 2\right),(2,3)}^{*}$.

and

$$
M(p, m)_{\lambda, \mu}=(-1)^{\ell(\lambda)+\ell(\mu)} \frac{\omega\left(B_{\mu, \lambda}\right)}{z_{\lambda}}
$$

For the proof of part (i) of Theorem 1, note that

$$
M(h, m)=M(h, p) M(p, m)
$$

and hence

$$
\begin{aligned}
M(h, m)_{\lambda, \mu} & =\sum_{\nu \vdash n} M(h, p)_{\lambda, \nu} M(p, m)_{\nu, \mu} \\
& =\sum_{\nu \vdash n}(-1)^{\ell(\lambda)+\ell(\nu)} \omega\left(B_{\lambda, \nu}\right)(-1)^{\ell(\nu)+\ell(\mu)} \frac{\omega\left(B_{\mu, \nu}\right)}{z_{\nu}} \\
& =\frac{(-1)^{\ell(\lambda)+\ell(\mu)}}{n !} \sum_{\nu \vdash n} \frac{n !}{z_{\nu}} \omega\left(B_{\lambda, \nu}\right) \omega\left(B_{\mu, \nu}\right) .
\end{aligned}
$$

Next we want to give a combinatorial interpretation to $\sum_{\nu \vdash n} \frac{n !}{z_{\nu}} \omega\left(B_{\lambda, \nu}\right) \omega\left(B_{\mu, \nu}\right)$. We let $\mathcal{B}_{\lambda, \mu}^{*}$ denote the set of $\lambda$ brick tabloids of shape $\mu$ where we mark one cell in the last brick of each row with an $*$. It is easy to see that $\omega\left(B_{\lambda, \mu}\right)=\left|\mathcal{B}_{\lambda, \mu}^{*}\right|$ since each $T \in \mathcal{B}_{\lambda, \mu}$ gives rise to $\omega(T)$ elements of $\mathcal{B}_{\lambda, \mu}^{*}$. For example, the $\lambda$-brick tabloid $T_{1}$ pictured in Figure 2 with $\omega\left(T_{1}\right)=2$ gives rise to the two tabloids in $\mathcal{B}_{\lambda, \mu}^{*}$ pictured in Figure 3.

Thus,

$$
\sum_{\nu \vdash n} \frac{n !}{z_{\nu}} \omega\left(B_{\lambda, \nu}\right) \omega\left(B_{\mu, \nu}\right)=\sum_{\nu \vdash n}\left|\mathcal{C}_{\nu} \times \mathcal{B}_{\lambda, \nu}^{*} \times \mathcal{B}_{\mu, \nu}^{*}\right| .
$$

Next we shall describe how we can associate to each triple $\left(\sigma, B_{1}, B_{2}\right) \in \mathcal{C}_{\nu} \times \mathcal{B}_{\lambda, \nu}^{*} \times$ $\mathcal{B}_{\mu, \nu}^{*}$, a labeled sequence of primitive bi-brick cycles $\psi\left(\sigma, B_{1}, B_{2}\right)$. The construction of 


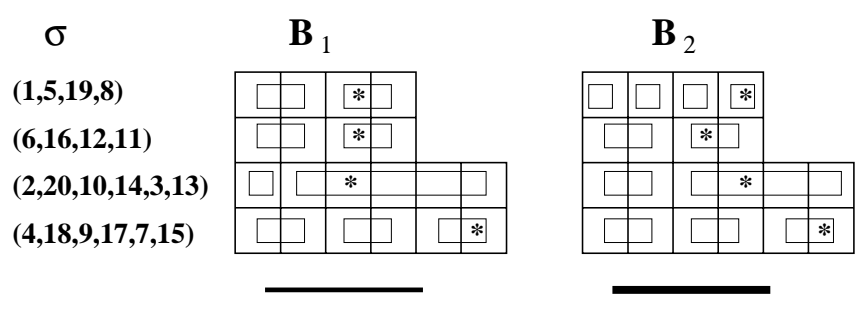

$\Theta\left(\sigma, \mathbf{B}_{1}, \mathbf{B}_{2}\right)=$
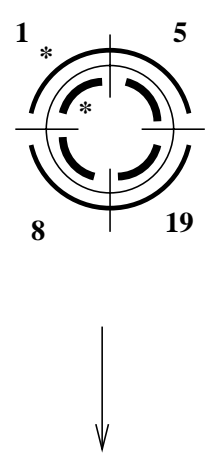

$\begin{array}{cccc}1 & 5 & 19 & 8 \\ \text { В } & \text { M } & \text { В } & \text { M }\end{array}$
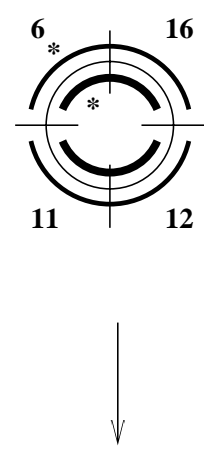

6161211 B N B N
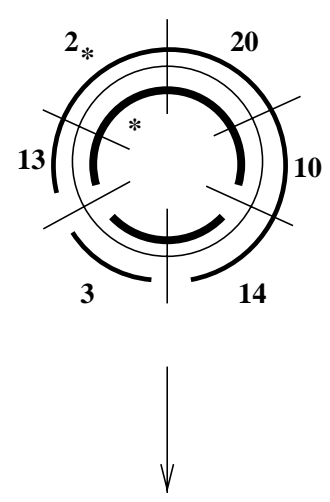

$\begin{array}{llllll}13 & 2 & 20 & 10 & 14 & 3 \\ \text { B } & \text { N } & \text { N } & \text { N } & \text { M } & \text { L }\end{array}$
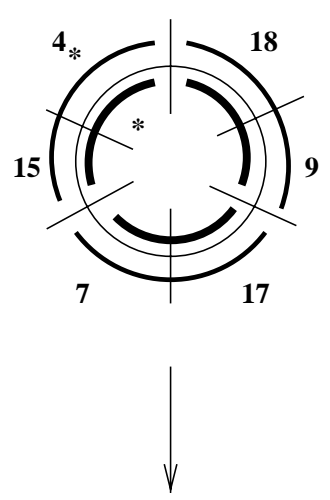

$\begin{array}{llllll}15 & 18 & 9 & 17 & 7\end{array}$

B N B N B N

Figure 9: $\Theta\left(\sigma, B_{1}, B_{2}\right)$.

$\psi\left(\sigma, B_{1}, B_{2}\right)$ is best described by referring to an example. Let $\lambda=\left(1,2^{7}, 5\right), \mu=\left(1^{4}, 2^{6}, 4\right)$, and $\nu=\left(4^{2}, 6^{2}\right)$.

We start with a triple $\left(\sigma, B_{1}, B_{2}\right) \in \mathcal{C}_{\nu} \times \mathcal{B}_{\lambda, \nu}^{*} \times \mathcal{B}_{\mu, \nu}^{*}$ as pictured at the top of Figure 4 . Each cycle $c$ of $\sigma$ is associated to a row of $B_{1}$ and $B_{2}$ of the same size as $c$. If there is more than one cycle of size $i$ in $\sigma$, then we list the cycles of $\sigma$ of size $i$ in increasing order according to their smallest elements, say $c_{1}^{i}, c_{2}^{i}, \ldots, c_{k_{i}}^{i}$. Then $c_{1}^{i}, \ldots, c_{k_{i}}^{i}$ are associated with the rows of size $i$ in $B_{1}$ and $B_{2}$ reading from top to bottom.

We then construct a bi-brick cycle out of each pair of corresponding rows of $B_{1}$ and $B_{2}$ by having the cells with $*$ 's correspond to the same cell in the bi-brick cycle. Next we label the bi-brick cycles with the elements of the corresponding cycle in $\sigma$ by having the smallest element of $\sigma$ correspond to the cell with the $*$ 's in the $\lambda$ and $\mu$ bricks in the bi-brick cycle. This process yields a labeled bi-brick permutation $\Theta\left(\sigma, B_{1}, B_{2}\right)$ as pictured in Figure 4. Note that since the smallest label corresponds to the cells with the $*$ 's, there is no loss in erasing the $*$ 's. Clearly we can use $\Theta\left(\sigma, B_{1}, B_{2}\right)$ to reconstruct, $\sigma, B_{1}$ and $B_{2}$ since we can (1) reconstruct the $*$ by picking the cell with the smallest label, (2) for each cycle, construct a pair of corresponding rows of $B_{1}$ and $B_{2}$ by placing the brick with the $*$ at the end of the row, and (3) order the rows of $B_{1}$ and $B_{2}$ of the same size by ensuring that the smallest elements in the corresponding cycles of $\sigma$ increase when we 


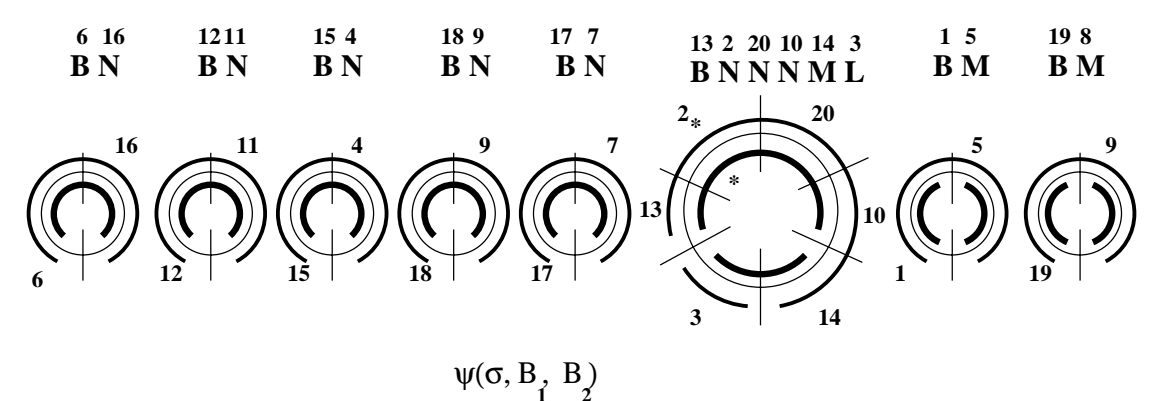

Figure 10: $\psi\left(\sigma, B_{1}, B_{2}\right)$.

read the cycles from top to bottom. Thus, $\Theta$ is a one-to-one correspondence between $\bigcup_{\nu} \mathcal{C}_{\nu} \times \mathcal{B}_{\lambda, \nu}^{*} \times \mathcal{B}_{\mu, \nu}^{*}$ and all labeled $(\lambda, \mu)$-bi-brick permutations.

Next we can replace each cycle by its word $W(C)$ and label $W(C)$ in the obvious manner to get a set of labeled words $\bar{W}\left(C_{1}\right), \ldots, \bar{W}\left(C_{k}\right)$ as pictured at the bottom of Figure 4. Now if the underlying word $W\left(C_{i}\right) \in\{B, L, M, N\}^{*}$ of $\bar{W}\left(C_{i}\right)$ factors into $\omega_{i}^{r_{i}}$ where $\omega_{i}$ is a Lyndon word, then we can factor $\bar{W}\left(C_{i}\right)$ into labeled Lyndon words $\bar{\omega}_{i, 1} \cdots \bar{\omega}_{i, r_{i}}$. The rotational symmetry of $C_{i}$ automatically ensures that $\omega_{i}$ corresponds to a primitive bi-brick cycle. We let $m_{i}$ denote the minimal label in $C_{i}$ and we cyclically arrange the labeled factors so that $m_{i}$ is a label in $\bar{\omega}_{i, 1}$. Now in this process, there may be more than one cycle that factors into a power of a given Lyndon word $u$. For example, in Figure 4, the second and fourth cycles factor into labeled Lyndon words whose underlying Lyndon word is $B N$. For any such Lyndon word $u$, let $C_{i_{1}}, \ldots, C_{i_{k}}$ be the set of cycles such that $\bar{W}\left(C_{i_{s}}\right)=\bar{u}_{1, i_{s}} \cdots \bar{u}_{t_{s}, i_{s}}$ where $m_{i_{1}}>\cdots>m_{i_{k}}$. This gives us a block of labeled words $\bar{u}_{1, i_{1}} \cdots \bar{u}_{t_{1}, i_{1}} \bar{u}_{1, i_{2}} \cdots \bar{u}_{t_{2}, i_{2}} \cdots \bar{u}_{1, i_{k}} \cdots \bar{u}_{t_{k}, i_{k}}=\vec{u}$ of labeled Lyndon words which all correspond to the same underlying Lyndon word $u$. Note that we easily reconstruct each $\bar{u}_{1, i_{j}} \cdots \bar{u}_{t_{j}, i_{j}}$ from $\vec{u}$ as follows. First by construction $\bar{u}_{1, i_{k}}$ is the labeled word with the smallest label in $\vec{u}$ so that $\bar{u}_{1, i_{k}} \cdots \bar{u}_{t_{k}, i_{k}}$ consists of the word with the smallest label in $\vec{u}$ together with all words of $\vec{u}$ to its right. Once we remove $\bar{u}_{1, i_{k}} \cdots \bar{u}_{t_{k}, i_{k}}$ from $\vec{u}$ to get $\vec{u}^{\prime}$, then $\bar{u}_{1, i_{k-1}}$ is the word with the smallest label in $\vec{u}^{\prime}$ so that $\bar{u}_{1, i_{k-1}} \cdots \bar{u}_{t_{k-1}, i_{k-1}}$ consists of the word of $\vec{u}^{\prime}$ with the smallest label in $\vec{u}^{\prime}$ together with all words to its right. Continuing on in this manner we can reconstruct $\bar{W}\left(C_{i_{1}}\right), \ldots, \bar{W}\left(C_{i_{k}}\right)$. Thus we have shown that each labeled $(\lambda, \mu)$-bi-brick permutation corresponds to a sequence of labeled Lyndon words where we order the blocks of labeled Lyndon words by the lexicographic order of their underlying Lyndon words as pictured in Figure 5. This sequence of labeled Lyndon words corresponds to the sequence of labeled primitive bi-brick cycles as pictured in Figure 4.

We call this sequence of labeled primitive bi-brick cycles $\psi\left(\sigma, B_{1}, B_{2}\right)$. The key point to observe is that the labels on the primitive cycles or, equivalently, on the sequence of Lyndon words is completely arbitrary since the reconstruction procedure described above will always produce a labeled $(\lambda, \mu)$-bi-brick permutation. It follows that each primitive $(\lambda, \mu)$-bi-brick permutation gives rise to $n$ ! labeled primitive $(\lambda, \mu)$-bi-brick permutations 
and hence to $n$ ! elements of $\bigcup_{\nu} \mathcal{C}_{\nu} \times \mathcal{B}_{\lambda, \nu}^{*} \times \mathcal{B}_{\mu, \nu}^{*}$. It thus follows that

$$
\sum_{\nu \vdash n}\left|\mathcal{C}_{\nu} \times \mathcal{B}_{\lambda, \nu}^{*} \times \mathcal{B}_{\mu, \nu}^{*}\right|=n !|P B(\lambda, \mu)|
$$

Combining (22), (23), and (24), we get that

$$
M(h, m)_{\lambda, \mu}=(-1)^{\ell(\lambda)+\ell(\mu)}|P B(\lambda, \mu)|
$$

as desired.

For part (ii) of Theorem 1, note that $M(e, m)=M(e, p) M(p, m)$ and hence

$$
\begin{aligned}
M(e, m)_{\lambda, \mu} & =\sum_{\nu \vdash n} M(e, p)_{\lambda, \nu} M(p, m)_{\nu, \mu} \\
& =\sum_{\nu \vdash n}(-1)^{n-\ell(\lambda)} \omega\left(B_{\lambda, \nu}\right)(-1)^{\ell(\nu)+\ell(\mu)} \frac{\omega\left(B_{\mu, \nu}\right)}{z_{\nu}} \\
& =\frac{(-1)^{\ell(\lambda)+\ell(\mu)}}{n !} \sum_{\nu \vdash n}(-1)^{n-\ell(\nu)} \frac{n !}{z_{\nu}} \omega\left(B_{\lambda, \nu}\right) \omega\left(B_{\mu, \nu}\right) \\
& =\frac{(-1)^{\ell(\lambda)+\ell(\mu)}}{n !} \sum_{\nu \vdash n}(-1)^{n-\ell(\nu)}\left|\mathcal{C}_{\nu} \times \mathcal{B}_{\lambda, \nu}^{*} \times \mathcal{B}_{\mu, \nu}^{*}\right| .
\end{aligned}
$$

We can then proceed exactly as in the proof of part (i) of Theorem 1 and associate to each triple $\left(\sigma, B_{1}, B_{2}\right)$ in $\bigcup_{\nu \vdash n} \mathcal{C}_{\nu} \times \mathcal{B}_{\lambda, \nu}^{*} \times \mathcal{B}_{\mu, \nu}^{*}$ a sequence of labeled primitive bi-brick cycles $\psi\left(\sigma, B_{1}, B_{2}\right)$ or, equivalently, a sequence of labeled Lyndon words $W\left(\psi\left(\sigma, B_{1}, B_{2}\right)\right)$. The only difference in this case is that $\psi\left(\sigma, B_{1}, B_{2}\right)$ carries a sign which is $(-1)^{n-c}$ where $c$ is the number of cycles of the labeled bi-brick permutation $\Theta\left(\sigma, B_{1}, B_{2}\right)$. We can define a simple sign reversing involution $f$ on the set of all such labeled sequences of Lyndon words $W\left(\psi\left(\sigma, B_{1}, B_{2}\right)\right)$ with $\left(\sigma, B_{1}, B_{2}\right) \in \bigcup_{\nu \vdash n} \mathcal{C}_{\nu} \times \mathcal{B}_{\lambda, \nu}^{*} \times \mathcal{B}_{\mu, \nu}^{*}$. That is, if the underlying bibrick permutation of $\psi\left(\sigma, B_{1}, B_{2}\right)$ is simple, we let $f\left(W\left(\psi\left(\sigma, B_{1}, B_{2}\right)\right)\right)=W\left(\psi\left(\sigma, B_{1}, B_{2}\right)\right)$. Otherwise, let $u$ be the lexicographically least word $v$ such that there are at least two occurrences labeled Lyndon words in $W\left(\psi\left(\sigma, B_{1}, B_{2}\right)\right)$ whose underlying Lyndon words is $v$. Let $\vec{u}$ be the block of all labeled Lyndon words in $W\left(\psi\left(\sigma, B_{1}, B_{2}\right)\right)$ whose underlying Lyndon words is $u$. We then define $f\left(W\left(\psi\left(\sigma, B_{1}, B_{2}\right)\right)\right.$ to be the labeled sequence of Lyndon words which results from interchanging the two labeled words in $\vec{u}$ with the two smallest minimal labels. For example, suppose that

$$
\vec{u}=\bar{u}_{1, i_{1}} \cdots \bar{u}_{t_{1}, i_{1}} \cdots \bar{u}_{1, i_{k-1}} \cdots \bar{u}_{t_{k-1}, i_{k-1}} \bar{u}_{1, i_{k}} \cdots \bar{u}_{t_{k}, i_{k}}
$$

is as described in our proof of part (i). Then $\bar{u}_{1, i_{k}}$ is the word with the smallest label. There are two possibilities for the word $\bar{u}$ whose minimal label is the next smallest. Namely either (a) $\bar{u}=\bar{u}_{1, i_{k-1}}$ if $\bar{u}$ occurs to the left of $\bar{u}_{1, i_{k}}$ or (b) $\bar{u}=\bar{u}_{j, i_{k}}$ with $j>1$ if $\bar{u}$ occurs to the right of $\bar{u}_{1, i_{k}}$. In case (a), $\vec{u}$ is replaced by

$$
\bar{u}_{1, i_{1}} \cdots \bar{u}_{t_{1}, i_{1}} \cdots \bar{u}_{1, i_{k-2}} \cdots \bar{u}_{t_{k-2}, i_{k-2}} \bar{u}_{1, i_{k}} \bar{u}_{2, i_{k-1}} \cdots \bar{u}_{t_{k-1}, i_{k-1}} \bar{u}_{1, i_{k-1}} \bar{u}_{2, i_{k}} \cdots \bar{u}_{t_{k}, i_{k}}
$$


in $f\left(W\left(\psi\left(\sigma, B_{1}, B_{2}\right)\right)\right)$. Now suppose that $\left(\sigma^{\prime}, B_{1}^{\prime}, B_{2}^{\prime}\right)$ is the triple such that $W\left(\psi\left(\sigma^{\prime}, B_{1}^{\prime}, B_{2}^{\prime}\right)\right)=f\left(W\left(\psi\left(\sigma, B_{1}, B_{2}\right)\right)\right)$. Then it easy to see that the sequence

$$
\bar{u}_{1, i_{k}} \bar{u}_{2, i_{k-1}} \cdots \bar{u}_{t_{k-1}, i_{k-1}} \bar{u}_{1, i_{k-1}} \bar{u}_{2, i_{k}} \cdots \bar{u}_{t_{k}, i_{k}}
$$

will be associated with a single cycle $C$ in $\Theta\left(\sigma^{\prime}, B_{1}^{\prime}, B_{2}^{\prime}\right)$ whereas the sequence

$$
\bar{u}_{1, i_{k-1}} \bar{u}_{2, i_{k-1}} \cdots \bar{u}_{t_{k-1}, i_{k-1}} \bar{u}_{1, i_{k}} \bar{u}_{2, i_{k}} \cdots \bar{u}_{t_{k}, i_{k}}
$$

produces two cycles in $\Theta\left(\sigma, B_{1}, B_{2}\right)$. In case (b), $\vec{u}$ is replaced by

$$
\bar{u}_{1, i_{1}} \cdots \bar{u}_{t_{1}, i_{1}} \cdots \bar{u}_{1, i_{k-1}} \cdots \bar{u}_{t_{k-1}, i_{k-1}} \bar{u}_{j, i_{k}} \bar{u}_{2, i_{k}} \cdots \bar{u}_{j-1, i_{k}}, \bar{u}_{1, i_{k}} \bar{u}_{j+1, i_{k}} \cdots \bar{u}_{t_{k}, i_{k}}
$$

in $f\left(W\left(\psi\left(\sigma, B_{1}, B_{2}\right)\right)\right)$. Again if $\left(\sigma^{\prime}, B_{1}^{\prime}, B_{2}^{\prime}\right)$ is the triple such that

$$
W\left(\psi\left(\left(\sigma^{\prime}, B_{1}^{\prime}, B_{2}^{\prime}\right)\right)\right)=f\left(W\left(\psi\left(\sigma, B_{1}, B_{2}\right)\right)\right)
$$

, then the sequence

$$
\bar{u}_{j, i_{k}} \bar{u}_{2, i_{k}} \cdots \bar{u}_{j-1, i_{k}} \bar{u}_{1, i_{k}} \bar{u}_{j+1, i_{k}} \cdots \bar{u}_{t_{k}, i_{k}}
$$

will be associated with two cycles in $\Theta\left(\sigma^{\prime}, B_{1}^{\prime}, B_{2}^{\prime}\right)$ whereas the sequence

$$
\bar{u}_{1, i_{k}} \bar{u}_{2, i_{k}} \cdots \bar{u}_{j-1, i_{k}} \bar{u}_{j, i_{k}} \bar{u}_{j+1, i_{k}} \cdots \bar{u}_{t_{k}, i_{k}}
$$

is associated to one cycle in $\Theta\left(\sigma, B_{1}, B_{2}\right)$. It follows that

$$
\operatorname{sgn}\left(\Theta\left(\left(\sigma, B_{1}, B_{2}\right)\right)=-\operatorname{sgn}\left(\Theta\left(\sigma^{\prime}, B_{1}^{\prime}, B_{2}^{\prime}\right)\right.\right.
$$

in both cases (a) and (b). For example, if we start with $\left.\left(\sigma, B_{1}, B_{2}\right)\right)$ of Figure 5 , then $\left(\sigma^{\prime}, B_{1}^{\prime}, B_{2}^{\prime}\right), f\left(W\left(\psi\left(\sigma, B_{1}, B_{2}\right)\right)\right)$, and $\Theta\left(\sigma^{\prime}, B_{1}^{\prime}, B_{2}^{\prime}\right)$ are pictured in Figure 6 .

Our involution $f$ shows that

$$
\begin{aligned}
& \frac{(-1)^{\ell(\lambda)+\ell(\mu)}}{n !} \sum_{\nu \vdash n}(-1)^{n-\ell(\nu)}\left|\mathcal{C}_{\nu} \times \mathcal{B}_{\lambda, \nu}^{*} \times \mathcal{B}_{\mu, \nu}^{*}\right|= \\
& \frac{(-1)^{\ell(\lambda)+\ell(\mu)}}{n !} \sum \operatorname{sgn}\left(\Theta\left(\sigma, B_{1}, B_{2}\right)\right)
\end{aligned}
$$

where the second sum runs over all $\left(\sigma, B_{1}, B_{2}\right)$ such that $W\left(\psi\left(\sigma, B_{1}, B_{2}\right)\right.$ has no repeated words or, equivalently, over all $\left(\sigma, B_{1}, B_{2}\right)$ such that underlying bi-brick permutation of $\Theta\left(\sigma, B_{1}, B_{2}\right)=\psi\left(\sigma, B_{1}, B_{2}\right)$ is simple. Once again, the labels on such labeled simple $(\lambda, \mu)$-bi-brick permutations are completely arbitrary so that each simple $(\lambda, \mu)$-bi-brick permutation gives rise to $n$ ! labeled simple $(\lambda, \mu)$-bi-brick permutations. Moreover, the signs of all these $n$ ! labeled simple bi-brick permutations are the same. Thus (25) and (26) imply that

$$
M(e, m)_{\lambda, \mu}=(-1)^{\ell(\lambda)+\ell(\mu)} \sum_{\theta \in S P B(\lambda, \mu)} \operatorname{sgn}(\theta) .
$$



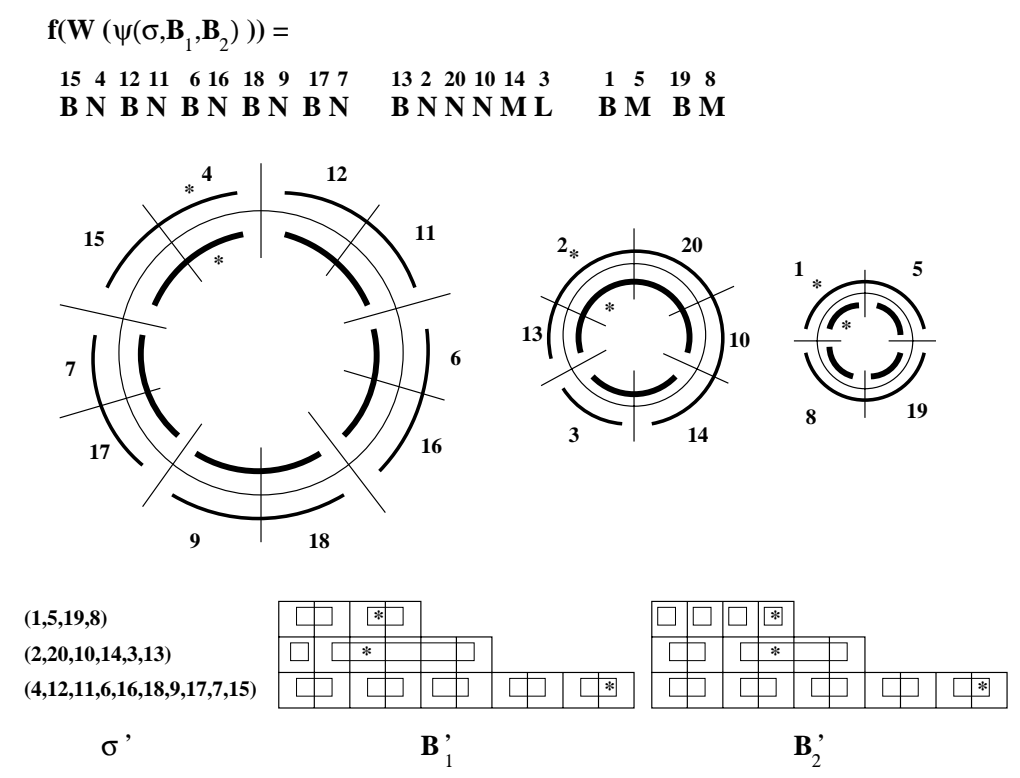

Figure 11: $f\left(W\left(\psi\left(\sigma, B_{1}, B_{2}\right)\right)\right)$.

\section{Further Involutions for the $M(e, m)_{\lambda, \mu}$}

In Section 2, we proved that

$$
M(e, m)_{\lambda, \mu}=(-1)^{\ell(\lambda)+\ell(\mu)} \sum_{\theta \in S P B(\lambda, \mu)} \operatorname{sgn}(\theta) .
$$

As we can see from our example in Figures 2-6, there is a considerable amount of cancellation that can occur in (28). In this section, we shall show that we can define further involutions on the set $S P B(\lambda, \mu)$ to explain some of this cancellation.

Recall that we can code each primitive bi-brick cycle by a Lyndon word over the alphabet $A=\{B, L, M, N\}$. Note that each bi-brick cycle $C$ has at least one $\lambda$-brick and at least one $\mu$-brick. Thus either (a) $W(C)$ must contain a $B$ if a $\lambda$-brick and $\mu$-brick start at the same cell or (b) $W(C)$ contains no $B$ but it does contain both an $L$ and $M$. Vice versa, it is easy to see that any word $w$ over $A$ which either (a) contains a $B$ or (b) contains no $B$ but does contain both an $L$ and a $M$ is of the form $W(C)$ for some bi-brick cycle $C$. Thus any simple primitive bi-brick permutation $\theta$ can be identified with a sequence of Lyndon words $W(\theta)=\left(w_{1}, \ldots, w_{p}\right)$ where $w_{1}<_{\ell} w_{2}<_{\ell} \cdots<_{\ell} w_{p}$ and $<_{\ell}$ denotes the lexicographic order relative to our ordering of the alphabet $B<L<N<M$. Moreover it must be the case that for all $1 \leq i \leq p$, either (a) $w_{i}$ contains a $B$ or (b) $w_{i}$ contains both an $L$ and an $M$ if $w_{i} \in\{L, N, M\}^{*}$. We let $\mathcal{S} \mathcal{L}$ denote the set of all such sequences of Lyndon words over the alphabet $A$. Given a sequence $\left(w_{1}, \ldots, w_{p}\right) \in \mathcal{S} \mathcal{L}$, we define the sign of $\left(w_{1}, \ldots, w_{p}\right), \operatorname{sgn}\left(w_{1}, \ldots, w_{p}\right)$, to be $(-1)^{\sum_{i=1}^{p}\left(\left|w_{i}\right|-1\right)}$. Thus if $\left(w_{1}, \ldots, w_{p}\right)=W(\theta)$ for some bi-brick permutation $\theta$, then $\operatorname{sgn}(\theta)=\operatorname{sgn}\left(w_{1}, \ldots, w_{p}\right)$. We shall define a series 
of sign reversing involutions on $\mathcal{S} \mathcal{L}$ which have the property that the collection of $\lambda$ and $\mu$ bricks in the corresponding simple primitive bi-brick permutations is preserved. These involutions will show that we can replace the sum on the right hand side of (28) by a more restricted sum. For example, let $\mathcal{S} \mathcal{L}_{B \leq 1}$ denote the set of all sequences of Lyndon words $\left(w_{1}, \ldots, w_{p}\right) \in \mathcal{S} \mathcal{L}$ such that $\left(w_{1}, \ldots w_{p}\right)$ contains at most one $B$. The sequences $\left(w_{1}, \ldots w_{p}\right) \in \mathcal{S L}_{B \leq 1}$ correspond to simple primitive bi-brick permutations $\theta$ such that as we traverse the cycles in a clockwise manner, there is at most one cell in $\theta$ which is the start of both a $\lambda$ and a $\mu$ brick. Our first result of this section will be to construct a sign reversing involution on $\mathcal{S} \mathcal{L}$ which proves the following.

\section{Theorem 2}

$$
M(e, m)_{\lambda, \mu}=(-1)^{\ell(\lambda)+\ell(\mu)} \sum_{\substack{\theta \in S P B(\lambda, \mu) \\ W(\theta) \in \mathcal{S} \mathcal{L}_{B \leq 1}}} \operatorname{sgn}(\theta) .
$$

Proof. Before we can define our desired involution on $\mathcal{S} \mathcal{L}$, we need to establish some notation. Let $X=\left\{x_{1}, \ldots, x_{n}\right\}$ be an ordered alphabet where $x_{1}<x_{2}<\cdots<x_{n}$. Let $X^{*}$ denote the set of all words over $X$ and $\operatorname{Lyn}(X)$ denote the set of all Lyndon words in $X^{*}$. Given $x \in X$, we let $x$-Lyn denote the set of all words in $\operatorname{Lyn}(X)$ which start with $x$. If $w=u v$ where $u, v \in X^{*}$, then we say $u$ is an initial segment of $w$ and write $u \sqsubseteq w$. If in addition, $|v| \geq 1$ and $|u| \geq 1$, then we say $u$ is a head of $w$ and $v$ is a tail of $w$. Recall that $<_{\ell}$ denotes the lexicographic order on $X^{*}$. We shall write $w<_{\ell} u$ if $w<_{\ell} u$ and $w \nsubseteq u$.

This given, we recall two characterizations of Lyndon words over $X$ which we shall use in our proofs which can be found in [5].

Lemma 1 (Proposition 5.1.2 in [5], page 65.) Let $w \in X^{*}$. Then $w \in \operatorname{Lyn}(X)$ if and only if $w<<_{\ell} v$ for any tail $v$ of $w$.

Lemma 2 (Proposition 5.1.3 in [5], page 66.)

Let $w \in X^{*}$. Then $w \in \operatorname{Lyn}(X)$ if and only if either (i) $w \in X$ or (ii) $w=u_{1} u_{2}$ where $u_{1}<_{\ell} u_{2}$ and $u_{1}, u_{2} \in \operatorname{Lyn}(X)$. In fact, if $w \in \operatorname{Lyn}(X),|w| \geq 2$, and $w=$ uv where $v$ is the longest tail of $w$ which is in $\operatorname{Lyn}(X)$, then $u \in \operatorname{Lyn}(X)$ and $u<_{\ell} w<_{\ell} v$.

This given, we define the following involution $I_{B}: \mathcal{S} \mathcal{L} \rightarrow \mathcal{S} \mathcal{L}$. Suppose $\left(w_{1}, \ldots, w_{t}\right) \in$ $\mathcal{S L}$ where $w_{1}<_{\ell} w_{2}<_{\ell} \cdots<_{\ell} w_{t}$. Let $m$ be the smallest $s \geq 0$ such that $w_{s+1} \notin B$-Lyn $(A)$ if there is such an $s$ and $m=t$ if $w_{t} \in B$ - Lyn $(A)$. Note that all words in $B$-Lyn $(A)$ are lexicographically less than the words in $\operatorname{Lyn}(A) \backslash B$ - $\operatorname{Lyn}(A)$. Hence it must be the case that $w_{m+1}, \ldots, w_{t} \in \operatorname{Lyn}(A) \backslash B$ - Lyn $(A)$. The definition of $I_{B}$ proceeds according to the following five cases.

Case $1 m=0$ so that no $B$ 's occur in $\left(w_{1}, \ldots, w_{t}\right)$. Then $I_{B}\left(w_{1}, \ldots, w_{t}\right)=\left(w_{1}, \ldots, w_{t}\right)$.

Case $2 m=1$ and $w_{1}$ contains exactly one $B$. Then $I_{B}\left(w_{1}, \ldots, w_{t}\right)=\left(w_{1}, \ldots, w_{t}\right)$. 


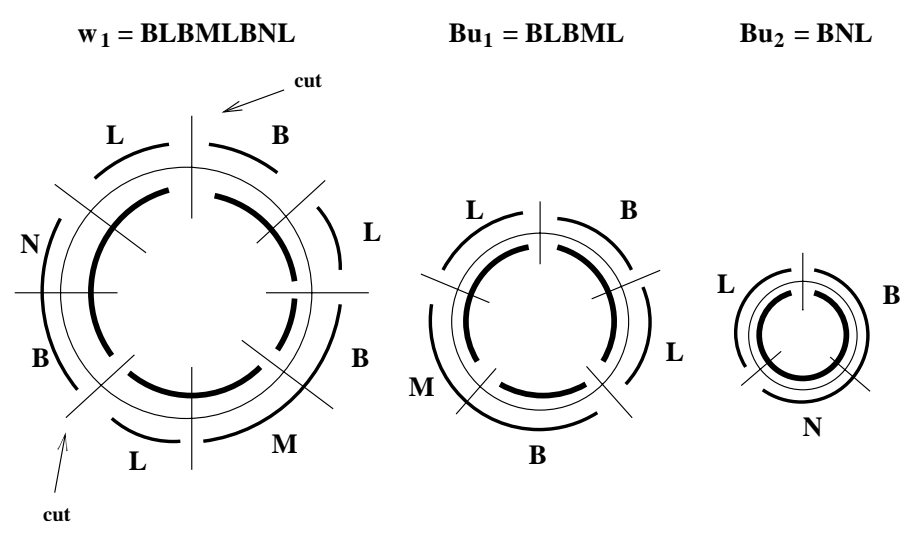

Figure 12: Cutting a bi-brick cycle at $B$ 's.

Case $3 m=1$ and $w_{1}$ contains two or more $B$ 's. Let $I_{B}\left(w_{1}, \ldots, w_{t}\right)=\left(u_{1}, v_{1}, w_{2}, \ldots, w_{t}\right)$ where $v_{1}$ is the shortest tail of $w_{1}$ such that $w_{1}=u_{1} v_{1}$ where $u_{1}, v_{1} \in B$ - Lyn $(A)$ and $u_{1}<_{\ell} v_{1}$.

Case $4 m>1$ and there is a tail $v$ of $w_{m}$ such that $w_{m}=u v$ where $u, v \in B-\operatorname{Lyn}(A)$ and $w_{m-1}<_{\ell} u<_{\ell} v$. Let $w_{m}=u_{m} v_{m}$ where $v_{m}$ is the shortest such tail $v$ of $w_{m}$ such that $w_{m}=u v, w_{m-1}<_{\ell} u<_{\ell} v$ and $u, v \in B$-Lyn $(A)$. We then set $I_{B}\left(w_{1}, \ldots, w_{t}\right)=$ $\left(w_{1}, \ldots w_{m-1}, u_{m}, v_{m}, w_{m+1}, \ldots, w_{t}\right)$.

Case $5 \quad m>1$ and not case 4 . Then set $I_{B}\left(w_{1}, \ldots, w_{t}\right)=$ $\left(w_{1}, \ldots w_{n-2}, w_{m-1} w_{m}, w_{m+1}, \ldots, w_{t}\right)$.

Before we proceed to show that $I_{B}$ is indeed a well defined involution, we pause to make a few remarks about the properties of $I_{B}$. First observe that if $w$ is the word of a bi-brick cycle $C$ and $w=B u_{1} B u_{2}$ where $u_{1}, u_{2} \in A^{*}$, then the bi-brick cycles $C_{1}$ and $C_{2}$ corresponding to $B u_{1}$ and $B u_{2}$ respectively can be constructed from $C$ by cutting $C$ at two cells which are the start of both $\lambda$ and $\mu$-bricks so that $C_{1}$ and $C_{2}$ contain the same $\lambda$ and $\mu$-bricks as $C$. See Figure 12 for an example. Thus if $\theta_{1} \in S P B(\lambda, \mu)$ is such that $W\left(\theta_{1}\right)=\left(w_{1}, \ldots, w_{t}\right)$, then there is a $\theta_{2} \in S P B(\lambda, \mu)$ such that $W\left(\theta_{2}\right)=I_{B}\left(w_{1}, \ldots, w_{t}\right)$.

Second we observe that if $\theta_{2}$ arises from $\theta_{1}$ by either splitting one cycle of $\theta_{1}$ into two cycles or combining two cycles of $\theta_{1}$ into one cycle, then $\operatorname{sgn}\left(\theta_{1}\right)=-\operatorname{sgn}\left(\theta_{2}\right)$. Thus once we have proved that $I_{B}$ is a well defined involution, it will follow that for any $n>0$ and partitions $\lambda$ and $\mu$ of $n$, 


$$
\begin{aligned}
\sum_{\theta \in S P B(\lambda, \mu)} \operatorname{sgn}(\theta) & =\sum_{\substack{\theta \in S P B(\lambda, \mu) \\
I_{B}(W(\theta))=W(\theta)}} \operatorname{sgn}(\theta) \\
& =\sum_{\substack{\theta \in S P B(\lambda, \mu) \\
W(\theta) \in \mathcal{S} \mathcal{L}_{B \leq 1}}} \operatorname{sgn}(\theta) .
\end{aligned}
$$

Thus Theorem 2 immediately follows once we have proved that $I_{B}$ is a well defined involution.

To see that $I_{B}$ is well defined, first consider Case 3. Thus $w_{1}$ is the only word in $\left(w_{1}, w_{2}, \ldots, w_{t}\right)$ which contains a $B$ and $w_{1}$ contains at least two $B$ 's. Thus we can write $w_{1}=B u_{1} B u_{2}$ where $u_{1} \in A^{*}$ and $u_{2} \in\{L, M, N\}^{*}$. It is easy to see that $B u_{2}$ has the property that every tail $v$ of $B u_{2}$ satisfies $B u_{2}<_{\ell} v$. Thus $B u_{2}$ is Lyndon by Lemma 1 . Now let $v^{\prime}$ be the longest tail $v$ of $w_{1}$ such that $v \in \operatorname{Lyn}(A)$. By Lemma 2, $w_{1}=u^{\prime} v^{\prime}$ where $u^{\prime}<_{\ell} v^{\prime}$ and $u^{\prime} \in B-\operatorname{Lyn}(A)$. Note that since $B u_{2}$ is a tail of $w_{1}$ in $\operatorname{Lyn}(A),\left|v^{\prime}\right| \geq\left|B u_{2}\right|$ so that $v^{\prime}$ must contain a $B$. But if $v^{\prime}$ contains a $B$, it must start with a $B$ since $v^{\prime} \in \operatorname{Lyn}(A)$. Thus $u^{\prime}, v^{\prime} \in B$-Lyn $(A)$ and $u^{\prime}<_{\ell} v^{\prime}$ so that $v^{\prime}$ is a candidate to be the $v_{1}$ of Case 3 . Hence $u_{1}$ and $v_{1}$ exist in Case 3. It easily follows that $I_{B}$ is well defined in all cases. Thus we need only show that $I_{B}$ is an involution.

To see that $I_{B}$ is an involution, first consider Case 3. Thus $v_{1}$ is the shortest tail $v$ of $w_{1}$ such that $w_{1}=u v$ where $u, v \in B-\operatorname{Lyn}(A)$ and $u<_{\ell} v$. We claim that it cannot be the case that $v_{1}=\alpha \beta$ where $|\alpha|,|\beta| \geq 1, \alpha, \beta \in B$-Lyn $(A)$ and $u_{1}<_{\ell} \alpha<_{\ell} \beta$. That is, if such $\alpha$ and $\beta$ exist, then $u_{1} \alpha \in B-\operatorname{Lyn}(A)$ by Lemma 2. But then $u_{1} \alpha<_{\ell} u_{1} \alpha \beta=w_{1}$ and $w_{1}<_{\ell} \beta$ by Lemma 1. Thus $u_{1} \alpha<_{\ell} \beta$ and $u_{1} \alpha, \beta \in B$-Lyn $(A)$ which would mean that $v_{1}$ is not the shortest tail $v$ of $w_{1}$ such that $w_{1}=u v$ where $u, v \in B-\operatorname{Lyn}(A)$ and $u<_{\ell} v$. It follows that there can be no such $\alpha$ and $\beta$ so that $\left(u_{1}, v_{1}, w_{2}, \ldots, w_{t}\right)$ is in Case 5 and hence $I_{B}\left(\left(u_{1}, v_{1}, w_{2}, \ldots, w_{t}\right)\right)=\left(w_{1}, \ldots, w_{t}\right)$. Similarly suppose that in Case $4, v_{m}=\alpha \beta$ where $|\alpha|,|\beta| \geq 1, \alpha, \beta \in B-\operatorname{Lyn}(A)$ and $v_{m-1}<_{\ell} \alpha<_{\ell} \beta$. Then $w_{m-1}<_{\ell} u_{m}<_{\ell} u_{m} \alpha<_{\ell} w_{m}<_{\ell}$ $\beta$ so that $w_{m-1}<_{\ell} u_{m} \alpha<_{\ell} \beta$. Again $u_{m} \alpha \in B$-Lyn $(A)$ by Lemma 2 so that $\beta$ would violate our choice of $v_{m}$ as the shortest tail $v$ of $w_{m}$ such that $w_{m}=u v$ where $u, v \in B$ $\operatorname{Lyn}(A)$ and $w_{m-1}<_{\ell} u<_{\ell} v$. Thus in Case $4,\left(w_{1}, \ldots, w_{m-1}, u_{m}, v_{m}, w_{m+1}, \ldots, w_{t}\right)$ is in Case 5 so that $I_{B}\left(\left(w_{1}, \ldots, w_{m-1}, u_{m}, v_{m}, w_{m+1}, \ldots, w_{t}\right)\right)=\left(w_{1}, \ldots, w_{t}\right)$.

Finally consider Case 5 . In this case, we must show that $w_{m}$ is the shortest tail $v$ of $w_{m-1} w_{m}$ such that $w_{m-1} w_{m}=u v$ where $u, v \in B$-Lyn $(A)$ and $w_{m-2}<_{\ell} u<_{\ell} v$. If not, there exists $\alpha, \beta \in A^{*}$ such that $w_{m}=\alpha \beta,|\alpha|,|\beta| \geq 1, \beta \in B-\operatorname{Lyn}(A), w_{m-1} \alpha \in B-\operatorname{Lyn}(A)$ and $w_{m-2}<_{\ell} w_{m-1} \alpha<_{\ell} \beta$. Assume $\beta$ is the longest possible tail of $w_{m}$ with this property. By Lemma 1, $w_{m-1} \alpha<_{\ell} \alpha$ and $w_{m}<_{\ell} \beta$. Thus $w_{m-1}<_{\ell} w_{m-1} \alpha<_{\ell} \alpha<_{\ell} w_{m}<_{\ell} \beta$ so that $w_{m-1}<_{\ell} \alpha<_{\ell} \beta$. Since $\beta \in B$ - Lyn $(A)$ and we are not in Case 4, we must conclude that $\alpha \notin \operatorname{Lyn}(A)$. Hence by Lemma 1 , there is a tail $v$ of $\alpha$ such that $v \leq_{\ell} \alpha$. Let $\delta$ be the shortest tail $v$ of $\alpha$ such that $v \leq_{\ell} \alpha$. Thus $\alpha=\gamma \delta$ where $\gamma, \delta \in A^{*}$ and $|\gamma|,|\delta| \geq 1$. It cannot be the case that $\delta<<_{\ell} \alpha$ since otherwise $\delta \beta$ is a tail of $w_{m}$ such 
that $\delta \beta<<_{\ell} \alpha \beta=w_{m}$ which would violate the fact that $w_{m} \in \operatorname{Lyn}(A)$. Thus $\delta \sqsubseteq \alpha$. We claim that $\delta \in B$ - $\operatorname{Lyn}(A)$ and $|\delta| \leq|\gamma|$. That is, if $\delta \notin \operatorname{Lyn}(A)$, then there is a tail $\theta$ of $\delta$ such that $\theta \leq_{\ell} \delta$. But then $\theta \leq_{\ell} \delta \leq_{\ell} \alpha$ so that $\theta$ would be a shorter tail $v$ of $\alpha$ such that $v \leq_{\ell} \alpha$ which violates our choice of $\delta$. Moreover, since $\alpha$ starts with a $B$, then $\delta$ must start with a $B$ and hence $\delta \in B$ - $\operatorname{Lyn}(A)$. If $|\gamma|<|\delta|$, then $\delta=\gamma \theta$ where $\theta \in A^{*}$. On the other hand, since $\delta \sqsubseteq \alpha, \alpha=\gamma \theta \psi$ for some $\psi \in A^{*}$. But then since $\alpha=\gamma \delta$, it must be the case that $\delta=\theta \psi$. But that would imply that $\theta$ is a tail of $\gamma \delta$ and $\theta \sqsubseteq \delta \sqsubseteq \gamma \delta=\alpha$ which would again violate our choice of $\delta$. Thus $|\delta| \leq|\gamma|$ as claimed. It follows that we can write $\alpha$ in the form $\alpha=\delta^{k} \xi \delta^{\ell}$ for some $k, \ell \geq 1$ where $\xi \in A^{*}$ is such that $\delta$ is neither a head nor tail of $\xi$. We note that it is possible that $\xi=\emptyset$ (the empty word), in which case, we assume $k=1$. Next observe that $\delta<_{\ell} \alpha<_{\ell} \alpha \beta=w_{m}$ and $w_{m}<_{\ell} \beta$ since $\beta$ is a tail of the Lyndon word $w_{m}$. Thus $\delta<_{\ell} \beta$. But then by Lemma $2, \delta \beta \in \operatorname{Lyn}(A)$. Hence $\delta<_{\ell} \delta \beta$ so that $\delta^{2} \beta \in \operatorname{Lyn}(A)$. Continuing on in this way, $\delta^{\ell} \beta \in \operatorname{Lyn}(A)$ and since $\delta$ starts with a $B, \delta^{\ell} \beta \in B$-Lyn $(A)$. But then $\delta^{\ell} \beta$ is a tail of $w_{m-1} w_{m}$ and $w_{m-1} w_{m} \in \operatorname{Lyn}(A)$ so that $w_{m-2}<_{\ell} w_{m-1}<_{\ell} w_{m-1} \delta^{k} \xi<_{\ell} w_{m-1} w_{m}<_{\ell} \delta^{\ell} \beta$. Hence $w_{m-2}<_{\ell} w_{m-1} \delta^{k} \xi<_{\ell} \delta^{\ell} \beta$. Our choice of $\beta$ forces us to conclude that $w_{m-1} \delta^{k} \xi \notin \operatorname{Lyn}(A)$. Thus there is a tail $\Theta$ of $w_{m-1} \delta^{k} \xi$ such that $\Theta \leq_{\ell} w_{m-1} \delta^{k} \xi$. We shall show that the existence of such a $\Theta$ leads to a contradiction so that there can be no such $\alpha$ and $\beta$. First observe that it cannot be that $\Theta<<_{\ell} w_{m-1} \delta^{k} \xi$ since otherwise $\Theta \delta^{\ell} \beta<<_{\ell} w_{m-1} \delta^{k} \xi \delta^{\ell} \beta=w_{m-1} w_{m}$ so that $\Theta \delta^{\ell} \beta$ would be a tail of $w_{m-1} w_{m}$ which is $\leq_{\ell} w_{m-1} w_{m}$. But $w_{m-1} w_{m} \in \operatorname{Lyn}(A)$ so there can be no such tail. Thus $\Theta \sqsubseteq w_{m-1} \delta^{k} \xi$. We now have three cases.

Case (i) $|\Theta|>\left|\delta^{k} \xi\right|$.

In this case $\Theta=\psi \delta^{k} \xi$ for some $\psi \in A^{*}$ with $|\psi| \geq 1$. Thus $\psi$ is a tail of $w_{m-1}$. On the other hand, $\Theta \sqsubseteq w_{m-1} \delta^{k} \xi$ so that $\psi$ is a head of $w_{m-1}$. Thus $\psi \leq_{\ell} w_{m-1}$ which violates the assumption that $w_{m-1} \in \operatorname{Lyn}(A)$.

Case (ii) $\left|\delta^{k} \xi\right| \geq|\Theta|>|\xi|$.

It follows that either (a) $\Theta=\delta^{j} \xi$ for some $1 \leq j \leq k$ or (b) $\Theta=\psi \delta^{j} \xi$ where $0 \leq j<k$ and $\psi$ is some tail of $\delta$. In case (a), $\delta$ would be both a head and a tail of $w_{m-1} \delta^{k} \xi \delta^{\ell}=w_{m-1} \alpha$ which would violate the fact that $w_{m-1} \alpha \in \operatorname{Lyn}(A)$. Similarly in case (b), $\psi$ would be both a tail and head of $w_{m-1} \alpha$ which again would violate our assumption that $w_{m-1} \alpha \in \operatorname{Lyn}(A)$. Thus Case (ii) cannot hold.

Case (iii) $|\xi| \geq|\Theta|$.

In this case $\Theta \sqsubseteq w_{m-1} \delta^{k} \xi<_{\ell} w_{m-1} \alpha<_{\ell} \delta$. Thus $\Theta<_{\ell} \delta$. It cannot be that $\Theta<<_{\ell} \delta$ since otherwise $\Theta \delta^{\ell} \beta$ is a tail of $w_{m}$ such that $\Theta \delta^{\ell} \beta<_{\ell} \delta \sqsubseteq \alpha \sqsubseteq w_{m}$ which would violate the fact that $w_{m} \in \operatorname{Lyn}(A)$. Thus it must be the case that $\Theta \sqsubseteq \delta$. It cannot be that $\Theta=\delta$ since then $\delta$ would be both a tail and a head of $w_{m-1} \delta^{k} \xi \delta^{\ell}=w_{m-1} \alpha$. Thus $\Theta$ is a head of $\delta$. Suppose that $\delta=\Theta \psi$ where $\psi \in A^{*}$ and $|\psi|=h \geq 1$. Next let $\delta=\eta \phi$ where $\eta, \phi \in A^{*}$ and $|\eta|=h$. Since $\delta \in \operatorname{Lyn}(A), \delta<_{\ell} \psi$ and, in fact, $\eta<<_{\ell} \psi$. Thus $\Theta \eta<<_{\ell} \Theta \psi$. But $\Theta \eta \sqsubseteq \Theta \delta^{\ell} \beta$ and $\Theta \delta^{\ell} \beta$ is a tail of $w_{m}$ while $\delta=\Theta \psi$ is a head of $w_{m}$. Thus $\Theta \delta^{\ell} \beta<<_{\ell} w_{m}$ which violates the fact that $w_{m} \in \operatorname{Lyn}(A)$. Thus case (iii) cannot hold. 
Thus in Case 5 , the assumption that there is a tail $\beta$ of $w_{m}$ such that $w_{m}=\alpha \beta$ and $w_{m-2}<_{\ell} w_{m-1} \alpha<_{\ell} \beta$ where $w_{m-1} \alpha, \beta \in B-\operatorname{Lyn}(A)$ leads to a contradiction. Thus $w_{m}$ is the shortest tail $v$ of $w_{m-1} w_{m}$ such that $w_{m-1} w_{m}=u v$ where $w_{m-2}<_{\ell} u<_{\ell} v$ and $u, v \in B$ - Lyn $(A)$. Hence in Case 5 , we can conclude that $I_{B}\left(\left(w_{1}, \ldots, w_{m-2}, w_{m-1} w_{m}\right.\right.$, $\left.\left.w_{m+1}, \ldots, w_{t}\right)\right)=\left(w_{1}, \ldots, w_{t}\right)$. Thus $I_{B}$ is a well defined involution as claimed.

It is not difficult to show that our next result is a consequence of the fact that $M(e, m)_{\lambda, \mu}=0$ if $\mu<_{D} \lambda^{\prime}$. However, we can use Theorem 2 to give a combinatorial proof of this result.

Theorem 3 If $\lambda$ and $\mu$ are partitions of $u$ and $\ell(\lambda)+\ell(\mu) \geq n+2$, then $M(e, m)_{\lambda, \mu}=0$.

Proof. Suppose that $\theta=\left(\theta_{1}, \ldots, \theta_{k}\right)$ is a simple primitive bi-brick permutation such that $W(\theta)=\left(W\left(\theta_{1}\right), \ldots, W\left(\theta_{k}\right)\right) \in \mathcal{S L}_{B \leq 1}$. For each $i$, suppose $\theta_{i}$ is a primitive bi-brick permutation of size $n_{i}$ of type $\left(\lambda^{(i)}, \mu^{(i)}\right)$. Thus $\lambda=\bigcup_{i=1}^{k} \lambda^{(i)}, \mu=\bigcup_{i=1}^{k} \mu^{(i)}, \ell(\lambda)=\sum_{i=1}^{k} \ell\left(\lambda^{(i)}\right)$, $\ell(\mu)=\sum_{i=1}^{k} \ell\left(\mu^{(i)}\right)$ and $n=\sum_{i=1}^{k} n_{i}$. Now $\ell\left(\lambda^{(i)}\right)$ is the number of cells of $\theta_{i}$ where a $\lambda$-brick starts and $\ell\left(\mu^{(i)}\right)$ is the number of cells of $\theta_{i}$ where a $\mu$-brick starts. It is easy to see that if $\ell\left(\lambda^{(i)}\right)+\ell\left(\mu^{(i)}\right) \geq n_{i}+2$, then there must be at least two cells of $\theta_{i}$ where both a $\lambda$-brick and a $\mu$-brick start and hence $W\left(\theta_{i}\right)$ would contain two $B$ 's. But by assumption, there is at most one $B$ in $W(\theta)$ and hence we can conclude that $\ell\left(\lambda^{(i)}\right)+\ell\left(\mu^{(i)}\right) \leq n_{i}+1$ for all $i$. If $\ell\left(\lambda^{(i)}\right)+\ell\left(\mu^{(i)}\right)=n_{i}+1$, then there must be at least one cell of $\theta_{i}$ where both a $\lambda$-brick and a $\mu$-brick start so that $W\left(\theta_{i}\right)$ will have at least one $B$. Thus if $W\left(\theta_{1}\right)<_{\ell} \cdots<_{\ell} W\left(\theta_{k}\right)$ and $W(\theta)$ has one $B$, then that $B$ must occur in $W\left(\theta_{1}\right)$. But then $\ell\left(\lambda^{(1)}\right)+\ell\left(\mu^{(1)}\right) \leq n_{1}+1$ and for all $j>1, \ell\left(\lambda^{(j)}\right)+\ell\left(\mu^{(j)}\right) \leq n_{j}$ since $W\left(\theta_{j}\right)$ has no $B$ 's. Thus $\ell(\lambda)+\ell(\mu)=\sum_{i=1}^{k} \ell\left(\lambda^{(i)}\right)+\ell\left(\mu^{(i)}\right) \leq n_{1}+1+\sum_{j=2}^{k} n_{i}=n+1$. By a similar argument we can show that if $W(\theta)$ has no $B$ 's, then $\ell(\lambda)+\ell(\mu) \leq n$. Thus if $\ell(\lambda)+\ell(\mu) \geq n+2$, there can be no simple primitive bi-brick permutations $\theta$ such that $W(\theta) \in \mathcal{S L}_{B \leq 1}$ and hence $M(e, m)_{\lambda, \mu}=0$ by Theorem 2 .

Next we shall consider involutions on primitive bi-brick permutations $\theta$ such that the word of $\theta, W(\theta)=\left(w_{1}, w_{2}, \ldots, w_{m}\right)$, does not contain a $B$. In this case every word $w_{i}$ must contain both an $L$ and an $M$. Recall that in our alphabet $L<N<M$. Thus each $w_{i}$ must have an initial segment of the form $\phi$ where $\phi \in L\{L, N\}^{*} M$. In fact, it is easy to see that $\phi$ must be a Lyndon word. That is, suppose for a contradiction that $\delta$ is a tail of $\phi$ such that $\delta \leq_{\ell} \phi$. Then $\delta=\alpha M$ where $\alpha<_{\ell} \phi$. That is, if $\alpha \sqsubseteq \phi$, then the $(|\alpha|+1)$ st letter of $\delta$, namely $M$, is greater than the $(|\alpha|+1)$ st letter of $\phi$ which is in $\{L, N\}$ so that $\phi<<_{\ell} \delta$. Since $\alpha<<_{\ell} \phi, w_{i}=\phi \beta$ where $\beta \in A^{*}$ and hence $w_{i}$ has a tail $\alpha M \beta$ such that $\alpha M \beta<<\phi \beta=w_{i}$. Thus $\phi$ must be a Lyndon word.

Given any Lyndon word $\phi \in L\{L, N\}^{*} M$, we say that a word $w \in A^{*}$ is $\phi$-Lyndon if $w \in \operatorname{Lyn}(A)$ and $\phi$ is an initial segment of $w$. We let $\phi-\operatorname{Lyn}(A)$ denote the set of all $\phi$-Lyndon words in $A^{*}$. Now suppose that $\psi$ and $\phi$ are Lyndon words in $L\{L, N\}^{*} M$ such 


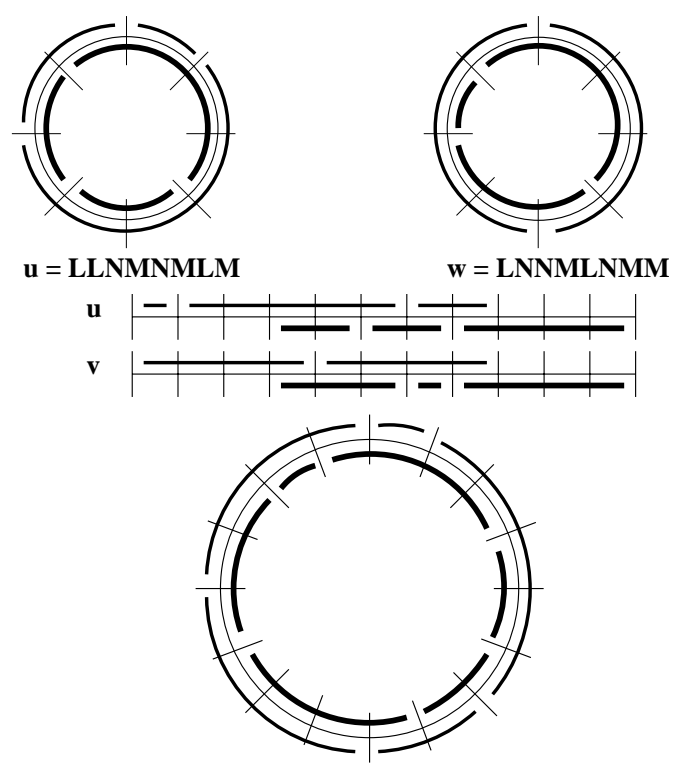

Figure 13: Combining two cycles arising from $\phi$-Lyndon words.

that $|\psi|=|\phi|, u$ is a $\psi$-Lyndon word, $w$ is a $\phi$-Lyndon word, and $u<_{\ell} w$. Moreover assume that $u$ is the word of some bi-brick cycle of type $(\alpha, \beta)$ and $w$ is the word of some bi-brick cycle of type $(\gamma, \delta)$. Then we claim $u w$ is also the word of a bi-brick cycle of type $(\alpha \cup \gamma, \beta \cup \delta)$. This is best explained by an example. Consider Figure 13. Here $\psi=L L N M, \phi=L N N M, u=L L N M N M L M$, and $w=L N N M L N M M$. Thus $\alpha=(1,2,5), \beta=(2,2,4), \gamma=(4,4)$, and $\delta=(1,3,4)$. It is then easy to see that we can break apart the cycle corresponding to $u$ and draw the two sets of bricks in a line so that the $\alpha$-bricks are on top starting with the bricks corresponding to the initial segment of $\psi$ of length $|\psi|-1$, i.e. $L L N$, and the $\beta$-bricks are on the bottom starting with the brick that corresponds with the $M$ of $\psi$. In this case since $|\psi|=4$, the $\alpha$-bricks will start $|\psi|-1=3$ squares ahead of the first $\beta$ brick and the last $\beta$ brick will extend 3 squares beyond the last $\alpha$-brick. Similarly, we can break apart the cycle corresponding to $w$ and draw the two sets of bricks so that the $\alpha$-bricks are on top starting with the bricks corresponding to the initial segment of $\phi$ of length $|\phi|-1$, i.e. $L N N$, and the $\delta$-bricks are on the bottom starting with the brick that corresponds with the $M$ of $\phi$. Again the $\gamma$-bricks will start 3 squares ahead of the first $\delta$-brick and the last $\delta$-brick will extend 3 squares beyond the last $\gamma$-brick. It is then easy to see that we can hook these two sequences together by having the $\gamma$-brick start immediately after the $\alpha$-bricks since the initial segment of 3 squares of $\gamma$-bricks fits over the 3 squares that the last $\beta$-brick extends beyond the last $\alpha$-brick. Then the combined sequence can be wrapped around a bi-brick cycle of length $|u w|$ so that the word of the bi-brick cycle is $u w$. Note that $u w$ must be the word of a primitive bi-brick cycle since $u w$ is in $\operatorname{Lyn}(A)$ by Lemma 2 .

This given, for any Lyndon word $\phi \in L\{L, N\}^{*} M$, we can define an involution $I_{\phi}$ : 
$\mathcal{S L} \rightarrow \mathcal{S L}$ in much the same way as we defined the involution $I_{B}$. That is, suppose that $\left(z_{1}, \ldots, z_{t}\right) \in \mathcal{S} \mathcal{L}$ where $z_{1}<_{\ell} \cdots<_{\ell} z_{t}$ and let $\left(w_{1}, \ldots, w_{m}\right)$ be the subsequence of $\left(z_{1}, \ldots, z_{t}\right)$ consisting of all words which are $\phi$-Lyndon. Then the definition of $I_{\phi}$ proceeds according to the following five cases.

Case 1. $m=0$ so that there are no $\phi$-Lyndon words in $\left(z_{1}, \ldots, z_{t}\right)$. Then $I_{\phi}\left(z_{1}, \ldots, z_{t}\right)=$ $\left(z_{1}, \ldots, z_{t}\right)$.

Case 2. $m=1$ and $w_{1}$ contains exactly one occurrence of $\phi$. (Here we say $\phi$ occurs in $w$ if $w=\alpha \phi \beta$ for some $\alpha, \beta \in A^{*}$.) Then $I_{\phi}\left(z_{1}, \ldots, z_{t}\right)=\left(z_{1}, \ldots, z_{t}\right)$.

Case 3. $m=1$ and $w_{1}$ contains two or more occurrences of $\phi$. Then let $v_{1}$ be the shortest tail of $w_{1}$ such that $w_{1}=u v$ where $u, v \in \phi$-Lyn $(A)$ and $u<_{\ell} v$. Assume $w_{1}=u_{1} v_{1}$ where $u_{1} \in \phi-\operatorname{Lyn}(A)$ and $u_{1}<_{\ell} v_{1}$. Then $I_{\phi}\left(z_{1}, \ldots, z_{t}\right)=\left(z_{1}^{\prime}, \ldots, z_{t+1}^{\prime}\right)$ where $z_{1}^{\prime}<_{\ell} \cdots<_{\ell} z_{t+1}^{\prime}$ is obtained from $\left(z_{1}, \ldots, z_{t}\right)$ by replacing the single word $w_{1}$ by two words $u_{1}$ and $v_{1}$.

Case 4. $m>1$ and there is a tail $v \in w_{m}$ such that $w_{m}=u v$ where $u, v \in \phi-\operatorname{Lyn}(A)$ and $w_{m-1}<_{\ell} u<_{\ell} v$. Then let $w_{m}=u_{m} v_{m}$ where $v_{m}$ is the shortest such tail of $w_{m}$ such that $w_{m-1}<_{\ell} u_{m}<_{\ell} v_{m}$ and $u_{m}, v_{m} \in \phi-\operatorname{Lyn}(A)$ and define $I_{\phi}\left(z_{1}, \ldots, z_{t}\right)=\left(z_{1}^{\prime}, \ldots, z_{t+1}^{\prime}\right)$ where $z_{1}^{\prime}<_{\ell} \cdots<_{\ell} z_{t+1}^{\prime}$ is obtained from $\left(z_{1}, \ldots, z_{t}\right)$ by replacing the single word $w_{m}$ by two words $u_{m}$ and $v_{m}$.

Case 5. $m>1$ and not case 4 . Then set $I_{\phi}\left(z_{1}, \ldots, z_{t}\right)=\left(z_{1}^{\prime}, \ldots, z_{t-1}^{\prime}\right)$ where $z_{1}^{\prime}<\cdots<$ $z_{t-1}^{\prime}$ is obtained from $\left(z_{1}, \ldots, z_{t}\right)$ by replacing the two words $w_{m-1}$ and $w_{m}$ by the single word $w_{m-1} w_{m}$.

The proof that $I_{\phi}$ is a well defined involution is almost word for word the same as the proof that $I_{B}$ is a well defined involution with two exceptions. That is, first we must show that in case $3, u_{1}$ and $v_{1}$ are defined and, second, we must show that in case 5 where the two words $w_{m-1}$ and $w_{m}$ get replaced by the single word $w_{m-1} w_{m}, w_{m}$ is the shortest $\phi$ Lyndon tail $v$ of $w_{m-1} w_{m}$ such that $w_{m-1} w_{m}=u v, u, v \in \phi-\operatorname{Lyn}(A)$ and $w_{m-2}<_{\ell} u<_{\ell} v$. That is, these are the only two places in the proof that $I_{B}$ is a well defined involution that we used any special properties of $B$-Lyndon words. Thus we shall only verify these two facts. First suppose that we are in case 3 and that $w_{1}$ has two occurrences of $\phi$. It is easy to see that since $\phi \in L\{L, N\}^{*} M$ that no two occurrences of $\phi$ in $w_{1}$ can overlap. Now consider the longest Lyndon tail $v$ of $w_{1}$. By Lemma 2, $w_{1}=u v$ where $u<_{\ell} v$ and $u, v \in \operatorname{Lyn}(A)$. We claim that $\phi$ occurs in $v$. That is, since there are two occurrences of $\phi$ in $w_{1}$, there is a tail $\beta$ of $w_{1}$ such that $\beta=\phi \gamma$ where there are no occurrences of $\phi$ in $\gamma$. We claim that $\beta$ is Lyndon. If not, $\beta=\alpha_{1} \alpha_{2}$ where $\alpha_{1}, \alpha_{2} \in A^{*}$ and $\phi \neq \alpha_{2} \leq_{\ell} \beta$. It cannot be that $\alpha_{2}<<_{\ell} \phi$ since otherwise $\alpha_{2}$ would be a tail of $w_{1}$ such that $\alpha_{2}<<_{\ell} \phi \sqsubseteq w_{1}$ violating the fact $w_{1} \in \operatorname{Lyn}(A)$. Similarly it cannot be that $\alpha_{2}$ is a head of $\phi$ since then $\alpha_{2}$ is a head of $w_{1}$ which again violates the fact that $w_{1} \in \operatorname{Lyn}(A)$. Thus it must be that $\phi \sqsubseteq \alpha_{2}$. But this is impossible because then $\beta$ has only one occurrence of $\phi$. Thus $\beta$ is Lyndon. But then since $v$ is the longest Lyndon tail of $w_{1}, \beta$ must be a final segment of $v$. We claim that this forces $\phi$ to be an initial segment of $v$. That is, if $v=\beta$, then certainly $\phi$ is an initial segment of $v$. If $v \neq \beta$, then $\beta$ is a tail of $v$ and hence $v<_{\ell} \beta$ since $v \in \operatorname{Lyn}(A)$. Since $\beta$ is a tail of $v$, it must be the case that $v<_{\ell} \beta$. However it cannot be that $v<<_{\ell} \phi$ since otherwise $v$ is a tail of $w_{1}$ such that $v<<_{\ell} \phi \sqsubseteq w_{1}$. Hence $\phi$ must be an initial segment of $v$. Thus $v$ is in $\phi-\operatorname{Lyn}(A)$. Since no two copies of $\phi$ can overlap 
in $w_{1}$, it must be the case that $v$ is a final segment of $\delta$ where $w_{1}=\phi \delta$. Thus if $w_{1}=u v$, then $\phi$ must be an initial segment of $u$. Hence by Lemma 2, $u<_{\ell} v$ and $u, v \in \operatorname{Lyn}(A)$ so that $u, v \in \phi-\operatorname{Lyn}(A)$. Hence there is at least one tail $v^{\prime}$ of $w_{1}$ such that $w_{1}=u^{\prime} v^{\prime}$, $u^{\prime}, v^{\prime} \in \phi-\operatorname{Lyn}(A)$ and $u^{\prime}<_{\ell} v^{\prime}$. Thus $I_{\phi}$ is defined in case 3 .

Next suppose that we are in case 5 . Thus we cannot write $w_{m}=u v$ where $u$ and $v$ are $\phi$-Lyndon words such that $w_{m-1}<_{\ell} u<_{\ell} v$. Now suppose there exist $\alpha, \beta$ such that $w_{m}=\alpha \beta,|\alpha|,|\beta| \geq 1, \beta$ and $w_{m-1} \alpha$ are $\phi$-Lyndon words, and $w_{m-2}<_{\ell} w_{m-1} \alpha<_{\ell} \beta$. Assume that $\beta$ is the longest possible tail of $w_{m}$ with this property. First observe that since $w_{m}=\alpha \beta$ and $\beta$ are $\phi$-Lyndon words and no two copies of $\phi$ in $w_{m}$ can overlap, $\phi$ must be an initial segment of $\alpha$. By Lemma $1, w_{m-1} \alpha<_{\ell} \alpha$ and $w_{m}<_{\ell} \beta$. Thus $w_{m-1}<_{\ell} w_{m-1} \alpha<_{\ell} \alpha<_{\ell} w_{m}<_{\ell} \beta$. Thus $w_{m-1}<_{\ell} \alpha<_{\ell} \beta$. Since $\beta \in \phi-\operatorname{Lyn}(A)$ and we are not in case 4 , we must conclude that $\alpha \notin \operatorname{Lyn}(A)$. By Lemma 1 , there is a tail $v$ of $\alpha$ such that $v \leq_{\ell} \alpha$. Pick $\delta$ to be the shortest tail $v$ of $\alpha$ such that $v \leq_{\ell} \alpha$ and write $\alpha=\gamma \delta$ where $\gamma, \delta \in A^{*}$ and $|\gamma|,|\delta| \geq 1$. It cannot be that $\delta<<_{\ell} \alpha$ since otherwise $\delta \beta$ is a tail of $w_{m}$ such that $\delta \beta<<_{\ell} \alpha \beta=w_{m}$ which would violate the fact that $w_{m} \in \operatorname{Lyn}(A)$. Thus $\delta \sqsubseteq \alpha$. We claim that $\delta \in \phi-\operatorname{Lyn}(A)$ and that $|\delta| \leq|\gamma|$. It cannot be that $\delta$ is an initial segment of $\phi$ since $\delta$ would then be both a tail and a head of $w_{m-1} \alpha$ which would violate the fact that $w_{m-1} \alpha \in \operatorname{Lyn}(A)$. Thus $\phi \sqsubseteq \delta$. Next suppose $\delta \notin \operatorname{Lyn}(A)$. Then there is a tail $\theta$ of $\delta$ such that $\theta \leq_{\ell} \delta$. But then $\theta$ is a tail of $\alpha$ such that $\theta \leq_{\ell} \delta \leq_{\ell} \alpha$ which would violate our choice of $\delta$. Thus $\delta \in \operatorname{Lyn}(A)$ and since $\phi \sqsubseteq \delta, \delta \in \phi-\operatorname{Lyn}(A)$. Next assume that $|\gamma|<|\delta|$. Thus $\delta=\gamma \theta$ where $\theta \in A^{*}$ and $|\theta| \geq 1$. But then $\alpha=\gamma \delta=\delta \psi$ for some $\psi \in A^{*}$ so that $\alpha=\gamma \theta \psi$. However, this would mean that $\delta=\gamma \theta=\theta \psi$ and hence $\theta$ would be both a head and a tail of $\delta$ which would violate the fact that $\delta \in \operatorname{Lyn}(A)$. Thus $\delta$ is a $\phi$-Lyndon word which is an initial segment of $\gamma$ as claimed. It follows that we can write $\alpha$ in the form $\alpha=\delta^{k} \xi \delta^{\ell}$ for some $k, \ell \geq 1$ where $\xi \in A^{*}$ and either $\xi=\emptyset$ or $\delta$ is neither a head nor a tail of $\xi$. We can now argue exactly as in the proof that $I_{B}$ is a well defined involution in case 5 that such a factorization leads to a contradiction. It follows that there can be no such $\beta$ and hence $w_{m}$ is the shortest $\phi$-Lyndon tail $v$ of $w_{m-1} w_{m}$ such that $w_{m-1} w_{m}=u v$ where $u, v \in \phi-\operatorname{Lyn}(A)$ and $w_{m-2}<_{\ell} u<_{\ell} v$.

We can thus conclude that $I_{\phi}$ is a well defined involution. Moreover if $I_{\phi}\left(z_{1}, \ldots, z_{t}\right) \neq$ $\left(z_{1}, \ldots, z_{t}\right)$, then there are simple primitive bi-brick permutations $\theta_{1}$ and $\theta_{2}$ of type $(\lambda, \mu)$ for some partitions $\lambda$ and $\mu$ such that $W\left(\theta_{1}\right)=\left(z_{1}, \ldots, z_{t}\right), W\left(\theta_{2}\right)=I_{\phi}\left(z_{1}, \ldots, z_{t}\right)$ and $\operatorname{sgn}\left(\theta_{1}\right)=-\operatorname{sgn}\left(\theta_{2}\right)$. Since $I_{\phi}$ affects only the $\phi$-Lyndon words in $\left(z_{1}, \ldots, z_{2}\right)$, we can apply the involutions $I_{B}$ and $I_{\phi}$ for all Lyndon words $\phi \in L\{L, N\}^{*} M$ sequentially to conclude the following.

Theorem 4 Let $\mathcal{S} \mathcal{L S}$ consist of all sequences of Lyndon words $\left(w_{1}, \ldots, w_{m}\right)$ such that $w_{1}<_{\ell} \cdots<_{\ell} w_{m},\left(w_{1}, \ldots, w_{m}\right)$ contains at most one $B$ and for any Lyndon word $\phi \in$ $L\{L, N\}^{*} M,\left(w_{1}, \ldots, w_{m}\right)$ contains at most one $\phi$-Lyndon word and if there is an $i$ such that $w_{i}$ is a $\phi$-Lyndon word, then there is exactly one occurrence of $\phi$ in $w_{i}$. Then for all $\lambda$ and $\mu$ which are partitions of $n$,

$$
M(e, m)_{\lambda, \mu}=(-1)^{\ell(\lambda)+\ell(\mu)} \sum_{\substack{\theta \in S P B(\lambda, \mu) \\ W(\theta) \in \mathcal{S} \mathcal{L} \mathcal{S}}} \operatorname{sgn}(\theta) .
$$


There are still more involutions that can be applied to the set of all $\theta \in \operatorname{SPB}(\lambda, \mu)$ with $W(\theta) \in \mathcal{S} \mathcal{L} \mathcal{S}$. We define a word $w$ to be $L$ - $s$-M-Lyndon if $w$ does not contain a $B$, $w$ has a head of the form $L \psi M$ where $\psi$ is a word of $\{L, N\}^{*}$ of length $s$ and $w$ has only one occurrence of $L \psi M$. Our observations above show that if $\alpha$ and $\beta$ are $L$-s- $M$-Lyndon words which come from primitive bi-brick cycles $\theta_{1}$ and $\theta_{2}$ and $\alpha<_{\ell} \beta$, then there is a primitive bi-brick cycle $\theta$ such that $\alpha \beta$ is the word of $\theta$. This suggests that we could define further involutions by combining $L$ - $s$ - $M$-Lyndon words. That is, suppose $\theta \in S P B(\lambda, \mu)$ with $W(\theta) \in \mathcal{S} \mathcal{L} \mathcal{S}$ and $W(\theta)=\left(w_{1}, \ldots, w_{m}\right)$ and $w_{i_{1}}<_{\ell} \cdots<_{\ell} w_{i_{k}}$ is the subsequence of $W(\theta)$ consisting of all $w_{i}$ which are $L$-s-M-Lyndon. Now suppose $k \geq 2$ and, for $1 \leq j \leq k, w_{i_{j}}$ is $\phi_{j}$-Lyndon where $\phi_{j}$ is a Lyndon word in $L\{L, N\}^{*} M$ of length $s+2$. If $\phi_{k-1}$ does not occur in $w_{i_{k}}$, then we know that we can combine the cycles corresponding to $w_{i_{k-1}}$ and $w_{i_{k}}$ into a single cycle $C$ such that $w(C)=w_{i_{k-1}} w_{i_{k}}$. Thus there will be a cycle $\theta^{\prime} \in S P B(\lambda, \mu)$ such that $W\left(\theta^{\prime}\right) \in \mathcal{S} \mathcal{L} \mathcal{S}, W\left(\theta^{\prime}\right)$ arises from $\theta$ by replacing the two words $w_{i_{k-1}}$ and $w_{i_{k}}$ by the single word $w_{i_{k-1}} w_{i_{k}}$ and $\operatorname{sgn}(\theta)=-\operatorname{sgn}\left(\theta^{\prime}\right)$. One could use this observation to try to construct an involution $I_{s}$ much like the involutions $I_{B}$ and $I_{\phi}$ described above. The problem is to find conditions which will allow us to recover $w_{i_{k-1}}$ and $w_{i_{k}}$ from $w_{i_{k-1}} w_{i_{k}}$. The following example will show that we cannot proceed exactly as before. That is, suppose that $s=5$ and our subsequence

$$
\left(w_{i_{1}}, \ldots, w_{i_{k}}\right)=\left(w_{1}, w_{2}\right)=(\operatorname{LLLLLLM,LLNLLNMLLNLNNNNNM).}
$$

There are three occurrences of seven letter Lyndon words in $L\{L, N\}^{*} M$ in $\left(w_{1}, w_{2}\right)$, namely $\phi_{1}=L L L L L L M, \phi_{2}=L L N L L N M$ and $\phi_{3}=L N N N N N M$. Note that we cannot break off $\phi_{3}$ from $w_{2}$ since $L L N L L N M L L N$ is not Lyndon. However if we combine $w_{1} w_{2}$, then we can break off $\phi_{3}$ from $w_{1} w_{2}$ since LLLLLLMLLNLLNMLLN is Lyndon.

Despite this example, one can define an involution $I_{s}$ for each $s \geq 0$ on the set of all $\theta \in S P B(\lambda, \mu)$ with $W(\theta) \in \mathcal{S} \mathcal{L} \mathcal{S}$ as follows. Let $\theta \in S P B(\lambda, \mu)$ and suppose $W(\theta)=\left(w_{1}, \ldots, w_{m}\right) \in \mathcal{S} \mathcal{L} \mathcal{S}$ and $w_{i_{1}}<_{\ell} \cdots<_{\ell} w_{i_{k}}$ is the subsequence of $W(\theta)$ consisting of all $w_{i}$ which are $L$-s-M-Lyndon. Let $w_{i_{j}}$ be $\phi_{j}$-Lyndon where $\phi_{j}$ is a Lyndon word in $L\{L, N\}^{*} M$ of length $s+2$ for $j=1, \ldots, k$. Let $\psi^{*}$ be the lexicographically largest $L-s$-M-Lyndon word which occurs as a subword in some $w_{i_{j}}$. We say that $w_{i_{k}}$ has a good $\psi^{*}$-tail if either (i) $\psi^{*}=\phi_{k}$ or (ii) $\phi_{k} \neq \psi^{*}$ and $w_{i_{k}}=\alpha \psi^{*} \beta$ where $\alpha$ is $\phi_{k}$-Lyndon and $\psi^{*} \beta$ is $\psi^{*}$-Lyndon and there is no occurrence of a $L-s-M$-Lyndon word in $\beta$. Observe that if $\phi_{k} \neq \psi^{*}$, then the good $\psi^{*}$-tail of $w_{i_{k}}$ is uniquely defined. The involution $I_{s}$ is defined as follows.

Case 1 If $\phi_{k}=\psi^{*}$ and $k \geq 2$, then $I_{s}(\theta)=\theta^{*}$ where $W\left(\theta^{*}\right)$ comes from $W(\theta)$ by replacing the two words $w_{i_{k-1}}$ and $w_{i_{k}}$ by a single word $w_{i_{k-1}} w_{i_{k}}$.

Note that in this case, $w_{i_{k}}$ does not contain any $L-s-M$-Lyndon subword other than the initial $\psi^{*}$. That is, by definition, $w_{i_{k}}$ has only one occurrence of $\psi^{*}$. Now if $\delta$ is another $L$-s-M-Lyndon occurring in $w_{i_{k}}$, then since $\delta$ and $\psi^{*}$ cannot overlap, it must be the case that $w_{i_{k}}=\psi^{*} \alpha \delta \beta$ for some $\alpha, \beta \in A^{*}$. But our choice of $\psi^{*}$ ensures that $\delta<<_{\ell} \psi^{*}$ so that $\delta \beta<<_{\ell} \psi^{*} \alpha \sqsubseteq w_{i_{k}}$ which would violate the fact that $w_{i_{k}}$ is Lyndon. It follows that $w_{i_{k-1}} w_{i_{k}}$ has only one occurrence of $\phi_{k-1}$ so that $W\left(\theta^{*}\right) \in \mathcal{S} \mathcal{L} \mathcal{S}$. Note also that in this 
case, $w_{i_{k}}$ is the good $\psi^{*}$-tail of $w_{i_{k-1}} w_{i_{k}}$.

Case 2 If $\psi^{*} \neq \phi_{k}$ and $w_{i_{k}}$ has a good $\psi^{*}$-tail $\beta$, then let $w_{i_{k}}=\alpha \beta$ and define $I_{s}(\theta)=\theta^{*}$ where $W\left(\theta^{*}\right)$ comes from $W(\theta)$ by replacing the $w_{i_{k}}$ by the two words $\alpha$ and $\beta$.

Note that if $k \geq 1$, then we have $\phi_{k-1}<<_{\ell} \phi_{k}<<_{\ell} \psi^{*}$ and hence $w_{i_{k-1}}<_{\ell} \alpha<_{\ell} \beta$.

It is easy to see that $I_{s}$ is an involution for each $s \geq 1$ and that we can apply these involutions independently. Note that there can be no such involution for $s=0$ because there is only one $L-0-M$-Lyndon word, namely, $L M$. Thus the fixed point set of all the involutions defined so far is the set $\operatorname{FSPB}(\lambda, \mu)$ consisting of $\theta \in S P B(\lambda, \mu)$ such that $W(\theta)=\left(w_{1}, \ldots, w_{m}\right)$ satisfies the following properties:

1. $\left(w_{1}, \ldots, w_{m}\right)$ contains at most one $B$,

2. for any Lyndon word $\phi \in L\{L, N\}^{*} M,\left(w_{1}, \ldots, w_{m}\right)$ contains at most one $\phi$-Lyndon word and if $w_{i}$ is a $\phi$-Lyndon word, then there is only one occurrence of $\phi$ in $w_{i}$, and

3. For each $s \geq 1$, if $w_{i_{1}}<_{\ell} \cdots<_{\ell} w_{i_{k}}$ is the subsequence of $W(\theta)$ consisting of all $w_{i}$ such that $w_{i}$ is $L$-s-M-Lyndon and, for all $j=1, \ldots, k, w_{i_{j}}$ is $\phi_{j}$-Lyndon where $\phi_{j}$ is a Lyndon word in $L\{L, N\}^{*} M$ of length $s+2$, and $\psi^{*}$ is the lexicographically largest $L$ - $s$ - $M$-Lyndon word which occurs in some $w_{i_{j}}$, then either (i) $\phi_{k}=\psi^{*}$ and $k=1$ or (ii) $\phi_{k} \neq \psi^{*}$ and $w_{i_{k}}$ does not have a good $\psi^{*}$-tail.

Thus we have the following.

Theorem 5 For all $n \geq 1$ and for all partitions, $\lambda$ and $\mu$ of $n$,

$$
M(e, m)_{\lambda, \mu}=(-1)^{\ell(\lambda)+\ell(\mu)} \sum_{\theta \in F S P B(\lambda, \mu)} \operatorname{sgn}(\theta) .
$$

\section{Some special cases of $M(h, m)_{\lambda, \mu}$ and $M(e, m)_{\lambda, \mu}$}

In this section, we shall apply Theorems $1-5$ to prove a few simple results about the values of $M(h, m)_{\lambda, \mu}$ and $M(e, m)_{\lambda, \mu}$ for certain classes of $\lambda$ and $\mu$. In particular, we shall give explicit formulas for $M(h, m)_{\lambda, \mu}$ and $M(e, m)_{\lambda, \mu}$ when $\lambda=\mu=\left(k^{n}\right)$ for some $k$ and $n$, when both $\lambda$ and $\mu$ are two-row shapes or when both $\lambda$ and $\mu$ are hook shapes. Finally we shall also find formulas $M(e, m)_{\lambda, \mu}$ when both $\lambda$ and $\mu$ are two-column shapes.

Theorem 6 For all $n \geq 1$ and $k \geq 1$,

$$
\begin{aligned}
M(h, m)_{\left(k^{n}\right),\left(k^{n}\right)} & =\left(\begin{array}{c}
n+k-1 \\
n
\end{array}\right) \text { and } \\
M(e, m)_{\left(k^{n}\right),\left(k^{n}\right)} & =(-1)^{n(k-1)}\left(\begin{array}{l}
k \\
n
\end{array}\right)
\end{aligned}
$$

where we set $\left(\begin{array}{l}k \\ n\end{array}\right)=0$ if $n>k$. 
Proof. It is easy to see that any bi-brick cycle of type $\left(\left(k^{p}\right),\left(k^{p}\right)\right)$ with $p \geq 2$ will have rotational symmetry. Thus the only primitive bi-brick cycles which contain only bricks of size $k$ must be of type $((k),(k))$. It is easy to see that there are exactly $k$ primitive bi-brick cycles of type $((k),(k))$. Thus any primitive bi-brick permutation of type $\left(\left(k^{n}\right),\left(k^{n}\right)\right)$ consists of $n$ cycles of type $((k),(k))$. Hence the number of primitive bibrick permutations of type $\left(\left(k^{n}\right),\left(k^{n}\right)\right)$ equals the number of non-negative integer valued solutions to $x_{1}+\cdots+x_{k}=n$ which is equal to $\left(\begin{array}{c}n+k-1 \\ n\end{array}\right)$. Thus $M(h, m)_{\left(k^{n}\right),\left(k^{n}\right)}=\left(\begin{array}{c}n+k-1 \\ n\end{array}\right)$.

A simple primitive bi-brick permutation of type $\left(\left(k^{n}\right),\left(k^{n}\right)\right)$ must consist of $n$ pairwise distinct primitive bi-brick cycles of type $((k),(k))$. Since there are $k$ primitive bi-brick cycles of type $((k),(k))$, there are $\left(\begin{array}{l}k \\ n\end{array}\right)$ simple primitive bi-brick permutations of type $\left(\left(k^{n}\right),\left(k^{n}\right)\right)$. Clearly the sign of any such simple primitive bi-brick permutation of type $\left(\left(k^{n}\right),\left(k^{n}\right)\right)$ is $(-1)^{n(k-1)}$ so that $M(e, m)_{\left(k^{n}\right),\left(k^{n}\right)}=(-1)^{n(k-1)}\left(\begin{array}{l}k \\ n\end{array}\right)$.

Next we shall give formulas for $M(h, m)_{\lambda, \mu}$ and $M(e, m)_{\lambda, \mu}$ when both $\lambda$ and $\mu$ are two-row shapes, i.e., when $\lambda=(a, b)$ and $\mu=(c, d)$ where $a+b=c+d=n$. Note that since both $M(h, m)_{\lambda, \mu}=M(h, m)_{\mu, \lambda}$ and $M(e, m)_{\lambda, \mu}=M(e, m)_{\mu, \lambda}$, there is no loss in generality in assuming that $a \leq c$.

Theorem 7 Suppose $\lambda=(a, b)$ and $\mu=(c, d)$ are two-part partitions of $n$ where $a \leq c$. Then

$$
\begin{aligned}
& M(h, m)_{\lambda, \mu}= \begin{cases}n & \text { if } a<c<d \\
n / 2 & \text { if } a<c=d \\
n+a b & \text { if } a=c<d \\
\left(\begin{array}{c}
a+1 \\
2
\end{array}\right) & \text { if } a=b=c=d\end{cases} \\
& M(e, m)_{\lambda, \mu}= \begin{cases}(-1)^{n-1} n & \text { if } a<c<d \\
(-1)^{n-1} n / 2 & \text { if } a<c=d \\
(-1)^{n-1}(n-a b) & \text { if } a=c<d \\
\left(\begin{array}{l}
a \\
2
\end{array}\right) & \text { if } a=b=c=d .\end{cases}
\end{aligned}
$$

Proof. This result easily follows from Theorem 1 once we make the following observations. First it is easy to see that if $a<c$, then the only primitive bi-brick permutation of type $((a, b),(c, d))$ consists of a single $n$-cycle. If $c<d$, there are clearly $n$ such primitive bi-brick cycles while if $c=d$, then there are $n / 2$ such primitive bi-brick cycles.

Next suppose $a=c<b=d$. Then there are $n$ primitive bi-brick cycles of size $n$ of type $((a, b),(c, d))$. The only other $((a, b),(a, b))$-primitive bi-brick permutations consist of two cycles, one of type $((a),(a))$ and the other of type $((b),(b))$. Clearly there are $a$ primitive bi-brick cycles of type $((a),(a))$ and there are $b$ primitive bi-brick cycles of type $((b),(b))$.

Finally if $a=b=c=d$, then our formulas follow from Theorem 6 .

Next we shall give formulas for $M(h, m)_{\lambda, \mu}$ and $M(e, m)_{\lambda, \mu}$ when both $\lambda$ and $\mu$ are hook shapes, i.e., when $\lambda=\left(1^{a}, b\right)$ and $\mu=\left(1^{c}, d\right)$ where $a+b=c+d=n$. Note that 
since both $M(h, m)_{\lambda, \mu}=M(h, m)_{\mu, \lambda}$ and $M(e, m)_{\lambda, \mu}=M(e, m)_{\mu, \lambda}$, there is no loss in generality in assuming that $b \leq d$.

Theorem 8 Let $\lambda=\left(1^{a}, b\right)$ and $\mu=\left(1^{c}, d\right)$ where $a+b=c+d=n$ and $b \leq d$.

1. If $d=n$ so that $\mu=(n)$, then

$$
M(h, m)_{\left(1^{a}, b\right),(n)}= \begin{cases}(-1)^{a} n & \text { if } b \geq 2 \\ (-1)^{n+1} & \text { if } b=1\end{cases}
$$

and

$$
M(e, m)_{\left(1^{a}, b\right),(n)}= \begin{cases}(-1)^{a+n-1} n & \text { if } b \geq 2 \\ 1 & \text { if } b=1 .\end{cases}
$$

2. If $b=1$ and $d \leq n-1$, then

$$
M(h, m)_{\left(1^{n}\right),\left(1^{c}, d\right)}= \begin{cases}(-1)^{n+c-1}(c+1) & \text { if } d \geq 2 \\ 1 & \text { if } d=1\end{cases}
$$

and

$$
M(e, m)_{\left(1^{n}\right),\left(1^{c}, d\right)}=0 .
$$

3. If $2 \leq b \leq d \leq n-1$, then

$$
M(h, m)_{\lambda, \mu}=(-1)^{a+c}\left((c+1) d+\left(\begin{array}{c}
c+1 \\
2
\end{array}\right)+\left(\begin{array}{c}
n-b-d+2 \\
2
\end{array}\right)\right)
$$

and

$$
M(e, m)_{\lambda, \mu}= \begin{cases}(-1)^{a+c+n+1} & \text { if } b+d \geq n+1 \\ 0 & \text { if } b+d \leq n .\end{cases}
$$

Proof. For (1), note that there are $n$ bi-brick cycles of type $\left(\left(1^{a}, b\right),(n)\right)$ if $b \geq 2$ depending on where the outside brick of size $b$ starts relative to the start of the inside brick of size $n$. Clearly all such bi-brick cycles are primitive. Thus $M(h, m)_{\left(1^{a}, b\right),(n)}=$ $(-1)^{\ell\left(\left(1^{a}, b\right)\right)+\ell((n))} n=(-1)^{a} n$ if $b \geq 2$. Since all such bi-brick cycles have sign $(-1)^{n-1}$, it follows that $M(e, m)_{\left(1^{a}, b\right),(n)}=(-1)^{a+n-1} n$ if $b \geq 2$.

In the case when $b=1$, there is a unique bi-brick cycle of type $\left(\left(1^{n}\right),(n)\right)$ which is also primitive. It then easily follows that

$$
\begin{aligned}
M(h, m)_{\left(1^{n}\right),(n)} & =(-1)^{\ell\left(\left(1^{n}\right)\right)+\ell((n))} 1=(-1)^{n+1} \text { and } \\
M(e, m)_{\left(1^{n}\right),(n)} & =(-1)^{\ell\left(\left(1^{n}\right)\right)+\ell((n))}(-1)^{n-1}=1 .
\end{aligned}
$$

For $(2)$, note that $M(e, m)_{\left(1^{n}\right),\left(1^{c}, d\right)}=0$ since $\left(1^{c}, d\right)<_{D}(n)$. One can also use Theorem 3 to conclude that $M(e, m)_{\left(1^{n}\right),\left(1^{c}, d\right)}=0$ since $\ell\left(\left(1^{n}\right)\right)+\ell\left(\left(1^{c}, d\right)\right) \geq n+2$. 
To compute $M(h, m)_{\left(1^{n}\right),\left(1^{c}, d\right)}$, first observe that if $d \geq 2$, then we can classify the primitive bi-brick permutations $\sigma$ of type $\left(\left(1^{n}\right),\left(1^{c}, d\right)\right)$ by the size of the bi-brick cycle $\theta$ which contains the brick of size $d$. That is, if $\theta$ is of type $\left(\left(1^{j+d}\right),\left(1^{j}, d\right)\right)$, then the rest of the bi-brick cycles of $\sigma$ must be of type $((1),(1))$ since the only primitive bi-brick cycle made up entirely of bricks of size 1 is of type $((1),(1))$. Moreover, $\theta$ is uniquely determined by $j$. Thus there is one primitive $\left(\left(1^{n}\right),\left(1^{c}, d\right)\right)$-bi-brick permutation for each $0 \leq j \leq c$. It easily follows that $M(h, m)_{\left(1^{n}\right),\left(1^{c}, d\right)}=(-1)^{n+c+1}(c+1)$ if $d \geq 2$. If $d=1$, there is a unique primitive bi-brick permutation of type $\left(\left(1^{n}\right),\left(1^{n}\right)\right)$ consisting of $n$ bi-brick cycles of type $((1),(1))$. Thus $M(h, m)_{\left(1^{n}\right),\left(1^{n}\right)}=(-1)^{n+n} 1=1$.

For (3), we observe that when $2 \leq b \leq d \leq n-1$, the primitive $\left(\left(1^{a}, b\right),\left(1^{c}, d\right)\right)$ bibrick permutations $\sigma$ fall into one of two categories. First, the bricks of size $b$ and $d$ can be in a single bi-brick cycle $\theta$ of type $\left(\left(1^{j+d-b}, b\right),\left(1^{j}, d\right)\right)$ for some $0 \leq j \leq c$ and the rest of $\sigma$ must consist of $c-j$ bi-brick cycles of type $((1),(1))$. Any bi-brick cycle $\theta$ of type $\left(\left(1^{j+d-b}, b\right),\left(1^{j}, d\right)\right)$ is automatically primitive since neither the inside bricks nor the outside bricks have rotational symmetry. Thus there are $d+j$ choices for $\theta$ depending on the relative placement of the brick of size $b$ with respect to the start of the brick of size $d$. Thus there are a total of $\sum_{j=0}^{c} d+j=(c+1) d+\left(\begin{array}{c}c+1 \\ 2\end{array}\right)$ primitive $\left(\left(1^{a}, b\right),\left(1^{c}, d\right)\right)$-bi-brick permutations where the brick of size $b$ and the brick of size $d$ lie in the same bi-brick cycle. The only other possibility is that the brick of size $b$ and the brick of size $d$ lie in different bi-brick cycles. Of course, this is only possible if $b+d \leq n$. In that case, a primitive bi-brick permutation $\sigma$ must consist of a bi-brick cycle $\theta_{1}$ of type $\left(\left(1^{x+d}\right),\left(1^{x}, d\right)\right)$, a bi-brick cycle $\theta_{2}$ of type $\left(\left(1^{y}, b\right),\left(1^{b+y}\right)\right)$ and $z$ bi-brick cycles of type $((1),(1))$ where $x+b+y+z=c=n-d$. Note that for fixed $x$ and $y$, the bi-brick cycles $\theta_{1}$ and $\theta_{2}$ are unique. It follows that the number of primitive $\left(\left(1^{a}, b\right),\left(1^{c}, d\right)\right)$-bi-brick permutations where the brick of size $b$ and the brick of size $d$ lie in different bi-brick cycles is the number of solutions of $x+y+z=n-b-d$ where $x, y, z \geq 0$. But clearly for each fixed $x$, there are $1+n-b-d-x$ choices for $y$ and $z$. Thus there there are a total of $\sum_{x=0}^{n-b-d} 1+n-b-d-x=\left(\begin{array}{c}n-b-d+2 \\ 2\end{array}\right)$ primitive $\left(\left(1^{a}, b\right),\left(1^{c}, d\right)\right)$-bi-brick permutations where the brick of size $b$ and the brick of size $d$ lie in different bi-brick cycles. Note that when $b+d>n$, then $\left(\begin{array}{c}n-b-d+2 \\ 2\end{array}\right)=0$ as it should be. Thus when $2 \leq b \leq d \leq n-1$, there are a total of $(c+1) d+\left(\begin{array}{c}c+1 \\ 2\end{array}\right)+\left(\begin{array}{c}n-b-d+2 \\ 2\end{array}\right)$ primitive $\left(\left(1^{a}, b\right),\left(1^{c}, d\right)\right)$-bi-brick permutations. Hence $M(h, m)_{\left(1^{a}, b\right),\left(1^{c}, d\right)}=(-1)^{a+c+2}\left((c+1) d+\left(\begin{array}{c}c+1 \\ 2\end{array}\right)+\left(\begin{array}{c}n-b-d+2 \\ 2\end{array}\right)\right)$ when $2 \leq b \leq d \leq n-1$. This proves (35).

Finally, we consider $M(e, m)_{\left(1^{a}, b\right),\left(1^{c}, d\right)}$ when $2 \leq b \leq d \leq n-1$. By Theorem 3 , $M(e, m)_{\left(1^{a}, b\right),\left(1^{c}, d\right)}=0$ if $a+c+2 \geq n+2$. But note that

$$
a+c+2 \geq n+2 \Longleftrightarrow n-b+n-d+2 \geq n+2 \Longleftrightarrow n \geq b+d .
$$

This means $M(e, m)_{\left(1^{a}, b\right),\left(1^{c}, d\right)}=0$ if $b+d \leq n$. Now suppose $b+d \geq n+1$. Clearly, in this case, a primitive $\left(\left(1^{a}, b\right),\left(1^{c}, d\right)\right)$-bi-brick permutation must have the brick of size $b$ and the brick of size $d$ in the same bi-brick cycle since we do not have enough room to have the brick of size $b$ and the brick of size $d$ in two different bi-brick cycles. By our analysis above, the only possible $\left(\left(1^{a}, b\right),\left(1^{c}, d\right)\right)$-bi-brick permutations consist of a primitive bi-brick cycle $\theta$ of type $\left(\left(1^{d-b+j}, b\right),\left(1^{j}, d\right)\right)$ plus $c-j$ bi-brick cycles of type $((1),(1))$. However only simple 
primitive bi-brick permutations contribute to $M(e, m)_{\lambda, \mu}$ so that we only have to analyze two types of $\left(\left(1^{a}, b\right),\left(1^{c}, d\right)\right)$-bi-brick permutations, namely, (i) the $\left(\left(1^{a}, b\right),\left(1^{c}, d\right)\right)$-bi-brick permutations $\sigma$ where there is one bi-brick cycle of type $((1),(1))$ and one bi-brick cycle of type $\left(\left(1^{a-1}, b\right),\left(1^{c-1}, d\right)\right)$ and (ii) the $\left(\left(1^{a}, b\right),\left(1^{c}, d\right)\right)$-bi-brick permutations $\tau$ where there is a single bi-brick cycle of type $\left(\left(1^{a}, b\right),\left(1^{c}, d\right)\right)$. Moreover, by Theorem 2 , we can assume that all such $\sigma$ and $\tau$ have the property that $W(\sigma), W(\tau) \in \mathcal{S} \mathcal{L}_{B \leq 1}$.

First consider the $\left(\left(1^{a}, b\right),\left(1^{c}, d\right)\right)$-bi-brick permutations $\sigma$ where there is one bi-brick cycle of type $((1),(1))$ and one bi-brick cycle of type $\left(\left(1^{a-1}, b\right),\left(1^{c-1}, d\right)\right)$ and $W(\sigma) \in$ $\mathcal{S} \mathcal{L}_{B \leq 1}$. Clearly the word of the bi-brick cycle of type $((1),(1))$ in $\sigma$ is $B$. Thus the bi-brick cycle $\theta$ of type $\left(\left(1^{a-1}, b\right),\left(1^{c-1}, d\right)\right)$ in $\sigma$ cannot have a $B$ in $W(\theta)$. This means that there cannot be two bricks in $\theta$ that start at the same cell. Note that since $c+d=n$ and we are assuming that $b+d \geq n+1$, it must be the case that $c<b$. Also $a \geq 1$ since $2 \leq b \leq d \leq n-1$. Let us draw the bi-brick cycle $\theta$ as pictured in Figure 14 where the outside brick of size $b$ starts in cell 1 at the top. We shall consider two cases, depending on whether $a>1$ or $a=1$. First suppose that $a>1$. Labeling the cells clockwise with $1, \ldots, n-1$ starting with cell 1 , we see that the outside of the cells $b+1, \ldots n-1$ following the end of the outside brick of size $b$ must be covered by bricks of size 1 . Thus an inside brick cannot start at any of the cells $b+1, \ldots, n-1,1$. It follows that the $c-1$ inside bricks must be cover a consecutive sequence of cells between cell 2 and cell $b-1$. If the $c-1$ inside bricks of size 1 end precisely at cell $b-1$, then they must start at cell $b-1-(c-2)=b-c+1$. Thus the start of the sequence of $c-1$ consecutive inside bricks of size 1 can start anywhere from cell 2 to cell $b-c+1$ and hence there are $b-c$ choices for $\theta$. In the case where $a=1$, then $b=n-1$ and the outside cells of $\theta$ are completely covered by the outside brick of size $b$. However, it still follows that the $c-1$ inside bricks must be cover a consecutive sequence of cells between cell 2 and cell $b-1$ so that there are $b-c$ choices for $\theta$ in this case as well. Thus the $\left(\left(1^{a}, b\right),\left(1^{c}, d\right)\right)$-bi-brick permutations $\sigma$ where there is one bi-brick cycle of type $((1),(1))$ and one bi-brick cycle of type $\left(\left(1^{a-1}, b\right),\left(1^{c-1}, d\right)\right)$ contribute a total of $(-1)^{a+c+2}(-1)^{n-2}(b-c)=(-1)^{a+c+n}(b-c)$ to $M(e, m)_{\left(1^{a}, b\right),\left(1^{c}, d\right)}$.

Finally consider the $\left(\left(1^{a}, b\right),\left(1^{c}, d\right)\right)$-bi-brick permutations $\tau$ where there is a single bi-brick cycle $\theta$ of type $\left(\left(1^{a}, b\right),\left(1^{c}, d\right)\right)$ and $W(\theta) \in \mathcal{S} \mathcal{L}_{B \leq 1}$. Hence there cannot be two cells $c$ in $\theta$ in which both an outside and an inside brick start at $c$. Again let us draw the bi-brick cycle $\theta$ as pictured in Figure 14 where the outside brick of size $b$ starts in cell 1 and we label the cells clockwise starting a cell 1 . Thus the outside of the cells $b+1, \ldots, n$ are covered by outside bricks of size 1 . We claim that the inside brick of size $d$ cannot start in cell 1 . That is, if the outside brick of size $d$ starts at cell 1 , then cell 1 contributes a $B$ to $W(\theta)$. But then the inside of cell $d+1$ must be covered by an inside brick of size 1 since $d \leq n-1$. But since $b \leq d$, the outside of cell $d+1$ must be covered by an outside brick of size 1 and hence cell $d+1$ would also contribute a $B$ to $W(\theta)$ which would violate our assumption that $W(\theta)$ has at most one $B$. Similarly the inside brick of size $d$ cannot start at any cells $b+2, \ldots, n$. That is, suppose the inside brick of size $d$ starts at cell $c_{1}$ where $b+1<c_{1} \leq n$. Then the cell $b+1$ must be covered by an inside brick of size 1 so that cell $b+1$ would contribute a $B$ to $W(\theta)$. But cell $c_{1}$ is covered by a outside brick of size 1 and 


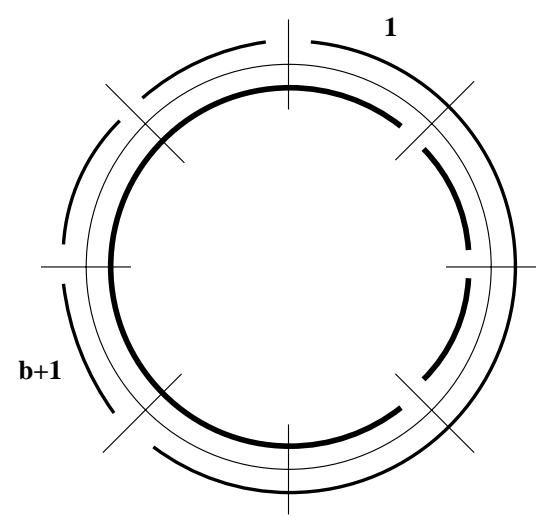

Figure 14: A bi-brick cycle $\theta$ of type $\left(\left(1^{a-1}, b\right),\left(1^{c-1}, d\right)\right)$ such that $W(\theta)$ has no $B$ 's.

hence cell $c_{1}$ would contribute a second $B$ to $W(\theta)$. Thus the inside brick of size $d$ must start somewhere between cell 2 and cell $b+1$. An alternative way to say this is that if we consider the block of $c$ inside bricks of size 1 read in a clockwise manner, then it must be the case that this block ends in one of the cells $1, \ldots, b$. We claim that the block of $c$ inside bricks of size 1 cannot end in any of the cells $1, \ldots, c-1$ since otherwise the cell $n$ and cell 1 would be covered by inside bricks of size 1 and yet both the cells are the start of outside bricks which would imply that $W(\theta)$ contain two $B$ 's. It follows that the block of $c$ inside bricks can end in cells $c, c+1, \ldots, b$ and hence there are $b-c+1$ possibilities for $\theta$. Thus the $\left(1^{a}, b\right),\left(1^{c}, d\right)$-bi-brick permutations $\sigma$ where there is a single bi-brick cycle of type $\left(\left(1^{a}, b\right),\left(1^{c}, d\right)\right)$ contribute a total of $(-1)^{a+c+2}(-1)^{n-1}(b-c+1)=(-1)^{a+c+n+1}(b-c+1)$ to $M(e, m)_{\left(1^{a}, b\right),\left(1^{c}, d\right)}$. Hence we have shown that

$$
\begin{aligned}
M(e, m)_{\left(1^{a}, b\right),\left(1^{c}, d\right)} & =(-1)^{a+c+n+1}(b-c+1)+(-1)^{a+c+n}(b-c) \\
& =(-1)^{a+c+n+1} .
\end{aligned}
$$

Our final result will show that if $\lambda$ and $\mu$ are partitions of $n$ with two or fewer columns, i.e. partitions of the form $\left(1^{s}, 2^{t}\right)$, then $M(e, m)_{\lambda, \mu}=0$ if $m \geq 5$.

Theorem 9 Suppose $\lambda=\left(1^{a}, 2^{b}\right)$ and $\mu=\left(1^{c}, 2^{d}\right)$ are partitions of $n$. Then

(1) if $n=2 s$ is even, then $M(e, m)_{\lambda, \mu}=0$ unless $\lambda=\mu=\left(2^{s}\right)$ or $\{\lambda, \mu\}=\left\{\left(1^{2}, 2^{s-1}\right),\left(2^{s}\right)\right\}$,

(2) if $n=2 s+1$ is odd, then $M(e, m)_{\lambda, \mu}=0$ unless $\lambda=\mu=\left(1,2^{s}\right)$,

(3) $M(e, m)_{\left(2^{s}\right),\left(2^{s}\right)}= \begin{cases}-2 & \text { if } s=1 \\ 1 & \text { if } s=2 \\ 0 & \text { if } s>2\end{cases}$ 
(4) $M(e, m)_{\left(1^{2}, 2^{s-1}\right),\left(2^{s}\right)}= \begin{cases}1, & \text { if } s=1 \\ 0 & \text { if } s \geq 2 .\end{cases}$

(5) $M(e, m)_{\left(1,2^{s}\right),\left(1,2^{s}\right)}= \begin{cases}1 & \text { if } s \leq 1, \\ 0 & \text { if } s>1 .\end{cases}$

Proof. Parts (1) and (2) follow immediately from Theorem 3. That is, if $n=2 s$, the condition $\ell(\lambda)+\ell(\mu) \leq n+1=2 s+1$ is satisfied only when $\lambda=\mu=\left(2^{s}\right)$ or when $\{\lambda, \mu\}=\left\{\left(1^{2}, 2^{s-1}\right),\left(2^{s}\right)\right\}$. Similarly when $n=2 s+1$, the condition $\ell(\lambda)+\ell(\mu) \leq n+1=$ $2 s+2$ is satisfied only when $\lambda=\mu=\left(1,2^{s}\right)$. Part (3) is just a special case of Theorem 6 .

For part (4), it is easy to check that there is one primitive bi-brick permutation of type $\left(\left(1^{2}\right),(2)\right.$ whose sign is -1 so that $M(e, m)_{\left(1^{2}\right),(2)}=1$. If $s \geq 2$, then $\left(2^{s}\right)<_{D}$ $(s-1, s+1)=\left(1^{2}, 2^{s-1}\right)^{\prime}$ so that $M(e, m)_{\left(1^{2}, 2^{s-1}\right),\left(2^{s}\right)}=0$. Similarly for part (5), it is easy to check by direct calculation that $M(e, m)_{(1),(1)}=1$ and $M(e, m)_{(1,2),(1,2)}=1$. For $s \geq 2$, $\left(1,2^{s}\right)<_{D}(s, s+1)=\left(1,2^{s}\right)^{\prime}$ so that $M_{\left(1,2^{s}\right),\left(1,2^{s}\right)}=0$.

\section{References}

[1] D. A. Beck, J. B. Remmel, and T. Whitehead, The combinatorics of transition matrices between the bases of the symmetric functions and the $B_{n}$ analogues, Discrete Mathematics 153, (1996), 3-27.

[2] P. Doubilet, Symmetric functions through the theory of distribution and occupancy, On the Foundations of Combinatorial Theory VII, Studies in Applied Math. Vol. 51, 1972.

[3] Ö. Ĕ̆gecioğlu and J. Remmel, Brick tabloids and the connection matrices between bases of symmetric functions, Discrete. Appl. Math. 34 (1991), 107-120.

[4] A. Kulikauskas, Ph.D. Thesis, Symmetric Functions of the Eigenvalues of a Matrix, University of California at San Diego, 1993.

[5] M. Lothaire, Combinatorics on Words, Addison Wesley, Reading, MA, 1983.

[6] I. G. Macdonald, Symmetric Functions and Hall Polynomials, 2nd ed., Oxford University Press, Oxford, 1995. 